\title{
The effects of radiative heat transfer during the melting process of a high temperature phase change material confined in a spherical shell
}

\author{
Antonio Ramos Archibold ${ }^{\mathrm{a}, \mathrm{b}, \mathrm{e}} \quad$ Muhammad M. Rahman $^{\mathrm{a}, \mathrm{b},{ }^{*}} \quad$ D. Yogi Goswami ${ }^{\mathrm{a}, \mathrm{c}} \quad$ Elias K. Stefanakos ${ }^{\mathrm{a}, \mathrm{d}}$ \\ ${ }^{a}$ Clean Energy Research Center, University of South Florida, Tampa, Fl, USA \\ ${ }^{b}$ Department of Mechanical Engineering, University of South Florida, Tampa, Fl, USA \\ ${ }^{c}$ Department of Chemical \& Biomedical Engineering, University of South Florida, Tampa, Fl, USA \\ ${ }^{d}$ Department of Electrical Engineering, University of South Florida, Tampa, Fl, USA \\ ${ }^{e}$ Department of Mechanical Engineering, Universidad Autónoma del Caribe, Barranquilla, Colombia
}

\begin{abstract}
The influence of radiation heat transfer during the phase change process of a storage material has been numerically analyzed in this study. Emphasis has been placed on the thermal characterization of a single constituent storage module rather than an entire storage system, in order to precisely capture the energy exchange contributions of all the fundamental heat transfer mechanisms during the melting of a phase change material (PCM) with tailored optical properties. The equations describing the conservation of mass, momentum and energy have been solved by using the control volume discretization approach, while the radiative transfer equation (RTE) was solved by the discrete ordinate method (DOM). The enthalpy-porosity method was used to track the PCM liquid/solid interface during the process. A parametric analysis has been performed in order to ascertain the effects of the optical thickness and the Planck, Grashof and Stefan numbers on the melting rate, as well as the total and radiative heat transfer rates at the inner surface of the shell. The results show that the presence of thermal radiation enhances the melting process. Correlations for the melt fraction and modified Nusselt number are developed for application in the design process of packed bed heat exchangers for latent heat thermal energy storage.
\end{abstract}

\section{Introduction}

The urgent need of sustainable and clean energy sources to secure the future energy production as well as expand the existing electricity generation market has motivated the rapid growth of commercial-scale central receiver concentrating solar power (CSP) plants during recent years [1,2]. Among the major subcomponents of the system namely, the heliostat field, the power conversion cycle and the receiver, the potential integration of thermal energy storage (TES) devices, during the operation of a CSP plant

\footnotetext{
* Corresponding author: Present address: Department of Mechanical Engineering, Wichita State University, Wichita, KS 67260, USA. Tel.:+ 1316-9786343, Fax: 1-316-9783236, e-mail: muhammad.rahman@wichita.edu (M M. Rahman)
} 
becomes extremely critical to dispatch power when solar irradiation is not available allowing the plant to operate in a cost-effective fashion and consequently reduce the plant levelized electricity cost. The current commercial TES option for CSP systems uses molten nitrate salts as the storage media while energy is stored via sensible heat $[3,4]$. In these systems, large amounts of storage material are used and, therefore, large tanks are required, thus increasing the overall cost of the system. In addition to the economic drawback, technical barriers associated with the low exergetic efficiency and material compatibility issues $[5,6]$ are key factors to encourage the near-term development of advanced thermal energy storage concepts. Numerous research and development initiatives have been directed towards the single tank thermocline system for sensible heat storage $[7,8,9]$ in an effort to reduce the cost of the TES system. However the primary challenge is the temperature degradation within the system, which results in lower exergetic efficiency [5]. In that context, the use of PCMs, for latent heat energy storage, seems to be one of the very promising methods because of its higher specific energy storage capacity which reduces the size and cost of the storage tank. The main drawbacks of PCMs for energy storage applications are low thermal conductivity, large volume expansion during melting [5] and chemical activity leading to corrosion. However, it is presumed that at elevated temperatures $\left(>800^{\circ} \mathrm{C}\right)$ radiative heat transfer becomes significant when compared to the others fundamental heat transfer modes. Consequently, the low thermal conductivity problem that penalizes the conduction energy transport during the melting and solidification processes can be overcome, particularly for transparent PCMs, by seeding the PCM with appropriate amounts of radiation absorbers. In this approach an infra-red transparent PCM with tailored absorptance for enhanced radiative transfer is encapsulated in several spherical shells with a highly emissive coating on the inner surface and use it in a packed bed heat exchanger. The objective of this study is to examine the energy exchange process during the melting of Sodium Chloride with enhanced radiative properties encapsulated in a spherical capsule at elevated temperatures. Among the different PCMs currently investigated as possible candidates to be used in thermal storage devices at elevated temperatures, $\mathrm{NaCl}$ has been selected in this investigation because of its high latent heat of fusion and relatively low cost. Also, its melting temperature $\left(800-802^{\circ} \mathrm{C}\right)$ matches with the typical operating range 62 of central receiver power plants.

63 The problem of heat transfer by radiation in a gray medium confined between two concentric black 64 spheres has been widely reported in the heat transfer literature. A simplified analytical method, based on a 65 diffusion approximation, for calculating radiation heat transfer in the aforementioned system has been 66 presented by Konakov [10]. Sparrow et al. [11] have discussed the contribution of the absorption 67 coefficient and the size of the container on the medium temperature distribution. In the model, an 68 absorbing-emitting, gray gas was assumed as a participating medium while uniform and equal 
volume through the gas was also considered. Numerical predictions were reported and the study concluded that the gas temperature variations are greater when the absorption coefficient increases for a fixed geometry. An approximate solution of the previously described problem has been reported by Dennar and Sibulkin [12] and Chou and Tien [13] based on an extension of the Milne-Eddington approximation (moment method) originally developed for plane layers. Tong and Swathi [14] evaluated the accuracy of the spherical harmonic numerical method by solving the same problem. In their model the scattering of radiation within the medium was considered and the influence of the differential approximation technique on the wall heat fluxes was presented. Ryhming [15], Viskanta and Crosbie [16] and Jia et al [17] extended the analysis of Sparrow et al [11] to include a uniform but different temperature at the bounded surfaces. It should be mentioned that in the results reported by [15] three values of inner to outer wall temperature ratios $T_{1} / T_{2}=2,5$ and 25 were reported. Viskanta and Merriam [18] performed a parametric investigation on the steady state conduction and radiation heating and cooling processes of an absorbing, emitting, scattering and gray medium enclosed in the space between two black, isothermal and concentric spheres. The influences of the Planck number, optical thickness, surface emissivities, inner to outer radius ratio, internal heat generation per unit volume and inner to outer wall temperature ratios were investigated. The study highlighted the strong influence of the surface emissivities on the local radiation heat transfer flux and reported an increase of the total heat flux at the inner surface of the system when the Planck number and the optical thickness decreases and increases respectively. The transient solution of the model reported by [18] has been obtained by Chu and Weng [19]. In the study, the equation of radiative transfer has been numerically solved by the spherical harmonic method. The transient cooling process by radiation of a stationary spherical gas mass has been analyzed by Viskanta and Lall [20]. The energy transfer by conduction and convection was neglected and a numerical solution of the energy equation has been obtained by the method of moments. The effect of the optical thickness of the gas on the local heat flux and temperature distribution has been presented. The Galerkin method was used by Thynell and Özisik [21] to solve the steady state condition of the problem reported by [20]. Tsai and Özisik [22] analyzed the transient combined conduction and radiation problem in an absorbing, emitting and isotropically scattering solid sphere subjected to a uniform temperature at the black boundary surface. The effects of the single scattering albedo and the radiation to conduction parameter on the temperature distribution and heat fluxes have been discussed. Siewert and Thomas [23] reported the steady state solution of the problem formulated by [22]. In the study the mathematical model was reduced based on a sphere to plane transformation technique and the spherical harmonics method was employed to numerically solve the reduced system. Bayazitoglu and Suryanarayana [24] numerically analyzed the influence of the Planck number and the optical thickness on the transient cooling process of a solid sphere surrounded by a gray, emitting, absorbing and isotropically scattering gas. The influence of 
anisotropic scattering on the combined conduction and radiation heat transfer on a spherical medium has been considered by Thynell [25]. Reported studies on the influence of thermal radiation in non-opaque media during phase change processes have been largely limited to the treatment of solidification in planar systems $[26,27,28]$, because of its simplicity and the fact that the contribution of natural convection can be justifiably ignored in this process $[29,30]$. In the present study, a concise description of the energy exchange interactions and induced fluid flow during the melting process of a PCM with enhanced radiative properties and encapsulated in a spherical shell is reported. The role played by all the fundamental heat transfer modes in the process is highlighted and analyzed. Except for the studies reported by $[31,32]$ for the combined mode heat transfer during melting in a rectangular and a square cavity respectively, no similar work has been reported in this field especially in curved media. The primary motivation of this investigation is to enlarge the limited literature available in the thermal characterization of PCM-based latent heat storage systems for concentrating solar power plants.

\section{Problem formulation and Mathematical model}

The PCM capsule is schematically shown in a cross sectional view in Figure 1. It consists of an opaque, gray and diffuse spherical shell of inner radius $R_{i}$ and wall thickness $\delta$. The space inside the shell is completely filled with a phase change material (PCM) which can conduct heat as well as absorb, emit and scatter radiant energy. Initially the PCM is in its solid state at a temperature $T_{o}$ and at time $t>0$, the outer boundary surface is subjected to a uniform temperature $T_{w}$ which is higher than the storage material melting temperature, $T_{m}$. The contribution of the radiant energy transfer within the PCM is characterized by the absorption and scattering coefficients $\kappa_{a}$ and $\sigma_{s}$ respectively, which in this study are independent of temperature and frequency. A uniform and wavelength independent PCM index of refraction $(n=1.4)$ has been considered in all the calculations. The following assumptions are considered: (1) The PCM is continuous, homogeneous and isotropic; (2) the PCM liquid phase is a viscous Newtonian fluid; (3) the flow is laminar, has no viscous dissipation and can be modeled as axisymmetric; (4) the whole system is initially sub-cooled at $790.7^{\circ} \mathrm{C}$; (5) The melting of $\mathrm{NaCl}$ takes place in the interval between $800.2^{\circ} \mathrm{C}$ and $800.7^{\circ} \mathrm{C}$, where the density in this interval varies linearly from $2160 \mathrm{~kg} / \mathrm{m}^{3}$ at $800.2^{\circ} \mathrm{C}$ to $1560 \mathrm{~kg} / \mathrm{m}^{3}$ at $800.7^{\circ} \mathrm{C}$, (6) In this study the PCM volumetric expansion due to melting has been neglected for simplicity, even though it is well known that the PCM volume changes can exceed $22 \%$ in response to the density variations during the phase change process. A rigorous model of the problem may consider a spherical capsule that is partially filled with solid PCM while the remaining volume is occupied by air. Also, it is clear that a convective boundary condition on the outer surface of the shell better characterizes the physical situation in a packed bed system. However, this study was mainly directed to analyze the simultaneous interaction of conduction, natural convection and thermal radiation and to quantify their 


$$
\frac{\partial \rho}{\partial t}+\frac{1}{r^{2}} \frac{\partial}{\partial r}\left(\rho v_{r} r^{2}\right)+\frac{1}{r \sin \theta} \frac{\partial}{\partial \theta}\left(\rho v_{\theta} \sin \theta\right)=0
$$

$$
\rho\left(\frac{\partial v_{r}}{\partial t}+v_{r} \frac{\partial v_{r}}{\partial r}+\frac{v_{\theta}}{r} \frac{\partial v_{r}}{\partial \theta}-\frac{v_{\theta}^{2}}{r}\right)=-\frac{\partial P}{\partial r}+\eta\left[\nabla^{2} v_{r}-\frac{2 v_{r}}{r^{2}}-\frac{2}{r^{2} \sin \theta} \frac{\partial\left(v_{\theta} \sin \theta\right)}{\partial \theta}-\frac{2 v_{\theta} \cot \theta}{r^{2}}\right]-\rho g_{r} \beta\left(T-T_{m}\right)
$$

$$
\rho\left(\frac{\partial v_{\theta}}{\partial t}+v_{r} \frac{\partial v_{\theta}}{\partial r}+\frac{v_{\theta}}{r} \frac{\partial v_{\theta}}{\partial \theta}+\frac{v_{r} v_{\theta}}{r}\right)=-\frac{1}{r} \frac{\partial P}{\partial r}+\eta\left[\nabla^{2} v_{\theta}+\frac{2}{r^{2}} \frac{\partial v_{r}}{\partial \theta}-\frac{v_{\theta}}{r^{2} \sin ^{2} \theta}\right]-\rho g_{\theta} \beta\left(T-T_{m}\right)
$$

Energy equation:

$$
\begin{aligned}
\frac{\partial h}{\partial t}+\frac{1}{r^{2}} \frac{\partial}{\partial r}\left(r^{2} v_{r} h\right) & +\frac{1}{r \sin \theta} \frac{\partial}{\partial \theta}\left(v_{\theta} \sin \theta h\right) \\
= & \alpha \nabla^{2} h-\frac{1}{\rho c_{p}}\left(\frac{\partial \lambda}{\partial t}+\frac{1}{r^{2}} \frac{\partial}{\partial r}\left(r^{2} v_{r} \lambda\right)+\frac{1}{r \sin \theta} \frac{\partial}{\partial \theta}\left(v_{\theta} \sin \theta \lambda\right)\right)-\nabla \cdot q_{r}
\end{aligned}
$$
medium can be expressed as [33]: 


$$
\frac{\mu}{r^{2}} \frac{\partial\left[r^{2} I(r, \mu)\right]}{\partial r}+\frac{1}{r} \frac{\partial\left[\left(1-\mu^{2}\right) I(r, \mu)\right]}{\partial \mu}+\left(\kappa_{a}+\sigma_{s}\right) I(r, \mu)=\kappa_{a} I_{b}(T)+\frac{\sigma_{s}}{2} \int_{-1}^{1} I\left(r, \mu^{\prime}\right) d \mu^{\prime}
$$

164

165

166

167

168

169

170

171

172

173

174

175

176

177

178

179

180

$$
q_{r}=2 \pi \int_{-1}^{1} I(r, \mu) \mu d \mu=2 \pi \sum_{i=1}^{n} \mu_{i} w_{i} I_{i}
$$

$\frac{\mu_{i}}{r^{2}} \frac{\partial\left[r^{2} I_{i}\right]}{\partial r}+\frac{1}{r} \frac{\partial\left[\left(1-\mu^{2}\right) I\right]_{\mu=\mu_{i}}}{\partial \mu}+\left(\kappa_{a}+\sigma_{s}\right) I_{i}=\kappa_{a} I_{b}+\frac{\sigma_{s}}{2} \sum_{i=1}^{n} w_{i} I_{i}$

where $I(r, \mu)$ is the radiation intensity, which is a function of the position and direction, $\mu$ is the cosine of the angle between the direction $\Omega$ of the beam and the extension of the radius vector $r, I_{b}(T)$ is the intensity of a black body radiation at the temperature of the medium. The last term on the right hand side of equation [5] represents the variation in radiation intensity, within the medium, due to scattering from the incoming direction $\mu^{\prime}$. In the discrete ordinate method, the RTE is approximated by discretizing the entire incident solid angle using a finite number of ordinate directions and corresponding weight factors $[34,35,36,37]$. Therefore, the integro-differential equation [5] is written for each ordinate and the integral term is replaced by a quadrature summed over each ordinate. Finally the equation reduces to a discrete system of differential equations, sufficient to solve for the radiation intensity $\left(I_{i}\right)$ at every location within the medium. The set of equations results in:

Where the subscript $\mathrm{i}$ denotes the quadrature points (ordinate directions) and $\mathrm{w}_{\mathrm{i}}$ is a weighting factor. Once the radiation intensity is known, the local radiative heat flux $\left(q_{r}\right)$ can be determined based on the following expression:

\section{Numerical Approach}

In order to solve the governing equations, the control volume technique, which employs an implicit scheme based on the SIMPLE (Semi-Implicit Method for Pressure-Linked Equations) algorithm [38], was used. Commercially available software Ansys/Fluent v12 was employed for the calculations. The second order upwind scheme was employed to discretize the momentum equations while the PRESTO (Pressure Staggering Option) scheme was adopted for pressure correction via the continuity equation. The energy equation was discretized using the First Order Upwind scheme. The under-relaxation factors for pressure, density and momentum were $0.4,0.7$, and 0.6 , respectively. Convergence of the solution was checked at each time step. Scaled absolute residuals of $10^{-4}, 10^{-3}$ and $10^{-6}$ were set as convergence criteria for 
continuity, velocity components and energy, respectively. The computational domain has been discretized based on a structured grid consisting of 8616 quadrilateral cells with a refined mesh distribution in the vicinity of the inner wall of the shell. Two different properties have been selected to evaluate the effect of the mesh grid size on the solution of the model. The mass average solid fraction and the dimensionless temperature $\left(\Theta_{n}\right)$ at the center point have been plotted as a function of the dimensionless time (see Figure 2) for different grid densities on the study case with $R_{i}=25 \mathrm{~mm}$ and $T_{w}-T_{m}=25^{\circ} \mathrm{C}$. From the solid fraction curves of Figure 2, it is observed that faster melting is predicted when the coarse grid is used. It is also shown that the solution tends to the same value when the element size decreases. Even though the grid independence test has been performed in the capsule with $R_{i}=0.025 \mathrm{~m}$, its validity can be extended to the study cases with different capsule sizes, because of the fact that the grid element size was kept approximately constant in all the calculations. To do that, the base computational domain (capsule with $R_{i}=0.025 \mathrm{~m}$ ) has been subdivided into five different zones which have been geometrically parameterized based on the shell dimensions and then used to estimate the amount of elements needed for the outer shell sizes. A grid distribution of 8616 cells, where the minimum and maximum element sizes are $0.33 \times 0.22 \mathrm{~mm}$ and $0.25 \times 0.56 \mathrm{~mm}$ (width $\mathrm{x}$ height) respectively, was found sufficient to achieve independence of the solution. The accuracy of the numerical approach implemented to solve the radiative heat transfer equation has been tested by solving the problem reported by Viskanta and Merriam [18]. The mathematical model was solved numerically using the control volume technique while the DOM [33] was used to solve the RTE. Numerically predicted dimensionless total $\left(\Psi_{n}\right)$ and radiative $\left(\Phi_{n}\right)$ heat flux at each surface of the system for different optical depths $\left(\tau_{2}\right)$ obtained from our simulations of the same problem are presented in Table 2. The effect of the inner sphere radius on the temperature distribution of the system is depicted in Figure 3. In the figure, the numerical solutions reported by [18] are also displayed and show a reasonably good agreement with the present results. Experiments were conducted to obtain the temperature response of $\mathrm{ACS}$ grade $\mathrm{NaCl}$ contained in a closed porcelain crucible during the sensible heating and phase change processes. A muffle furnace was used to thermally cycle the sample while continuous temperature measurements at the center of the sample and the wall of the porcelain crucible, were recorded. The numerical predictions have been validated by the experimental results, and reasonably good agreement was found. A detailed description of the experimental procedure and the comparison between the numerical and experimental results has been reported by Archibold et al. [39]

\section{Results and Discussion}

Ten different cases were analyzed in this study as summarized in Table 3. The effect of the optical thickness $(\tau)$ by changing the absorption coefficient $\left(\kappa_{a}\right)$, on the melting process for a constant capsule size is considered in cases 1 to 4 . It should be pointed out that the first case was used as a limiting case in 
which $\kappa_{a}=0$ and also zero emissivity $(\varepsilon)$ were assumed at the inner surface of the shell. It may be noticed that the Planck number $\left(P l=\kappa\left(\kappa_{a}+\sigma_{s}\right) / 4 n \sigma \bar{T}^{3}\right)$ also varies in these study cases. In cases 5 and 6 , the effects of the optical thickness and Grashof number (Gr) are analyzed by manipulating the capsule size. In the present model it was assumed that the mean beam length is the inner radius of the shell therefore the optical thickness can be calculated from the equation: $\tau=\left(\kappa_{a}+\sigma_{s}\right) R_{i}$, where $\sigma_{s}$ is the scattering coefficient. Cases 7 and 8 have been included in order to evaluate the combined influence of the Grashof and Stefan numbers on the thermal performance of the system, as the outer wall temperature $\left(T_{w}\right)$ changes. The effect of the scattering albedo $\left(\omega=\sigma_{s} /\left[\kappa_{a}+\sigma_{s}\right]\right)$ is analyzed in cases 9 and 10 . The present study is mainly directed to the analysis of the energy interactions that can occur in an attenuating and emitting thermal storage medium. The medium absorbs a fraction of the radiant energy from the inner shell wall due to photon/wave attenuation, and transforms it into thermal energy in the solid phase and consequently induces a faster melting process. It has been reported by [40] that the analyzed PCM, is mostly transparent to thermal radiation in the wavelength range of interest $(1.5 \leq \lambda \leq 9.0 \mu \mathrm{m})$, therefore an absorptive additive must be added to modify the PCM optical properties and improve the absorption of thermal radiation. In the present numerical model, it is assumed that the additive particles dissolve into the PCM to form a homogeneous and isotropic substance. Based on that, the effect of the additive concentration on the system can be represented by changing the absorption coefficient of the PCM, with the assumption that the higher the concentration the larger the absorption value. Figures 4 to 6 show the effect of $\kappa_{a}$ on different output variables of the system, for study cases 1 to 4 . From Figure 4 it can be observed that there is a faster melting with an increase of the absorption coefficient as compared to the limiting case of no radiation. A $10.4 \%$ decrease in the total melting time was observed in case $3\left(\kappa_{a}=\right.$ $\left.100 \mathrm{~m}^{-1}\right)$ as compared to case $1\left(\kappa_{a}=0.0 \mathrm{~m}^{-1}\right)$. A fraction of the incoming irradiation from the inner surface of the shell is attenuated by absorption, in this case, through the medium path length causing faster heating of the PCM and therefore faster melting of the solid PCM. The total and radiation heat transfer rates at the inner surface of the shell are shown in Figures 5 and 6. A closer look of the heat rate order of magnitude reveals that the contribution of the radiative heat to the total energy exchange is significantly smaller. This fact could explain the small difference found in the total melting time described previously. A possible explanation is that under the investigated thermal conditions $\left(T_{m} / T_{w}=\right.$ 0.977 for the large wall temperature study case) the contribution of thermal radiation does not play a first order role during the melting process as the energy transfer by natural convection does. Inspection of Figure 5 reveals that high heat transfer values are obtained initially because of the high temperature difference between the outer surface of the shell and the initial temperature of the system. Thermal energy is transported through the shell wall and the inward melting process begins with the creation of the first layer of molten PCM in the vicinity of the inner surface of the shell. The combined effect of the shell wall 
and molten PCM thermal resistances generates the sharp decrease on the heat transfer curves observed within the first 25-30sec in Figure 5. As the molten PCM layer gets larger, the contribution of natural convection (induced by the PCM liquid density gradient in the presence of gravity) becomes significant; consequently the heat transfer rate starts to increase and subsequently reaches a maximum (see the time period between $30 \mathrm{sec}$ to 1.0 minute, Figure 5). It should be noticed that the aforementioned events also characterize the case where the radiation within the medium is included; however, they occurred at a higher thermal energy level, indicating the contribution of radiant energy transfer on the net heat rate. After the maximum is attained, a slow-decay trend is observed in the curves toward the thermal equilibrium state. The net heat transfer rate at the inner surface of the shell was found to increase with the contribution of the participating radiation within the PCM. As seen from the curves, no significant difference between the heat transfer curves is found when the absorption coefficient changes from $100 \mathrm{~m}^{-1}$ to $200 \mathrm{~m}^{-1}$. The small difference in the radiant heat transfer of these two cases, observed in Figure 6, can be used to understand this trend. Since no additional improvements on the total energy flux at the capsule wall was observed with the absorption coefficient values higher than $100 \mathrm{~m}^{-1}$, the results for the case in which the absorption coefficient is $200 \mathrm{~m}^{-1}$ were not included in Figures 4 and 7 . Figure 7 depicts the average temperature of the center point of the capsule during the process. Each curve can be subdivided into three different zones: the solid phase sensible heating portion $\left(790.7^{\circ} \mathrm{C} \leq T \leq 800.2^{\circ} \mathrm{C}\right)$, the phase change $\left(800.2^{\circ} \mathrm{C} \leq T \leq 800.7^{\circ} \mathrm{C}\right)$ and liquid phase sensible heating $\left(800.7^{\circ} \mathrm{C} \leq T \leq 825.7^{\circ} \mathrm{C}\right)$. Special attention should be paid to the temperature response of the system during the solid phase heating. It can be observed that the time to reach $800.2^{\circ} \mathrm{C}$ at the center point is $78.6 \%$ faster in case $3\left(\kappa_{a}=\right.$ $100 \mathrm{~m}^{-1}$ ) as compared to the study case with no radiation. The results show that the presence of radiation during this period significantly enhances the heating process, based on the fact that, during this period the process is characterized by radiation and conduction heat transfer with radiation being the dominant heat transfer mode. Figure 8 illustrates the combined influence of the Grashof number and the optical thickness on the total heat transfer rate at the inner surface of the shell. Three different shell sizes have been analyzed $\left(R_{i}=20,25\right.$ and $\left.30 \mathrm{~mm}\right)$. As expected, by increasing the shell size, the total heat transfer rate increases. The larger the capsule, the larger the available heat transfer area for the energy transfer. Also, with the increase of the capsule size, the Grashof number increases as well, enhancing the contribution of natural convection. Simultaneously, the optical thickness of the medium becomes larger by increasing the path length over which radiation must travel. Under these conditions, the neighboring elements of the inner wall surface are mainly influenced by radiant energy interactions. Figure 9 shows the radiation heat transfer rate for the aforementioned cases. The same trend as that shown in Figure 8 is observed. An important observation between the figures is that, the radiation heat transfer curves exhibit a monotonically decreasing behavior which is characteristic of this type of process as the temperature 
gradient within the PCM decreases. The total heat transfer, which is mainly convective, sharply decreases at early times, then increases, reaching a maximum, and finally shows a slow decay trend toward thermal equilibrium. In order to graphically illustrate the process, the numerically predicted solid fraction, dimensionless streamlines and irradiation contours for case 5 are presented in composite diagrams illustrated in Figure 10 at different dimensionless times. The streamline contours are shown on the right half of each circle, whereas the irradiation contours are drawn on the left half, with the vertical axis of the sphere separating the two fields. The solid fraction distribution at the given instant is shown in the gray region of each plot. A dimensionless radial $(\mathrm{R})$ and axial $(\mathrm{Z})$ coordinate system has been used to locate each plot in the figure in order to provide a quantitative measure of the location and shape of the liquid/solid interface during the process, where the irradiation $\mathrm{G}$ is the rate at which radiating energy is incident on a location. It can be observed that the initial spherical shape of the solid fraction is converted to an oblate spheroid due to the buoyancy induced circulation flow that is formed in the PCM liquid phase located at the top portion of the container.

As the melting process proceeds, the remaining solid fraction reduces its size and remains at the bottom of the shell because of the density difference between the liquid and solid phases. A complete description of the events shown in Figure 10 and its physical interpretation has been provided in [41] and [42]. Inspection of the dimensionless streamline contour values reveals that, at the beginning of the process, the convective flow, which is characterized by a single circulating cell (called primary) reaches a maximum value (see Fig. 10b). It can be observed that after this time period, a secondary convection cell is developed at the top portion of the domain mainly induced by the primary (see Fig. 10c). As the process continues, more liquid PCM is created and the secondary circulating flow grows in physical size and strength while the primary shrinks in size and loses energy. The aforementioned trend is maintained during the dimensionless time period $4.62 \times 10^{-3} \leq$ SteFo $\leq 8.47 \times 10^{-3}$ (see Figs. 10d-10f). Figure $10 \mathrm{~g}$ shows that eventually both cells achieve the same energy level and merge into a single circulating pattern that characterizes the latest stages of the process (see Figs. 10h and 10i) which can be compared with the steady state solution of the natural convection problem of a fluid contained in a spherical shell. A welldefined crescent shaped circulation flow pattern can be observed with the circulation center close to the capsule wall.

Heretofore the discussion focuses on the output variables included on the right hand side of each plot of Figure 10, however valuable information about the irradiation distribution within the PCM during the process is presented on the left hand side of each plot. During the early periods, the irradiation distribution has a concentric ring shape, especially in the solid portion of the PCM (see Figs. 10a and 10b). From the dimensionless contour values it can be seen that the irradiation level rapidly increases within the domain as time proceeds (Fig. 10c). For dimensionless times higher than SteFo $=3.08 \times 10^{-3}$, 
when the natural convection has properly set, a stratified temperature field is formed with high temperature gradients near to the inner shell wall, especially in the lower part of the hemisphere [42], and, as seen in figure $10 \mathrm{~d}$ to $10 \mathrm{i}$ the irradiation follows the same trend.

The effect of the shell outer surface temperature was examined by analyzing cases 3, 7 and 8 . A $20 \mathrm{~mm}$ inner radius pellet was subjected to three different boundary condition values above the PCM melting temperature. The predicted liquid mass fraction of the aforementioned cases is depicted in Figure 11. As expected, faster melting was achieved with higher values of the shell outer wall temperature. The melting time was $20 \%$ shorter for Case $7\left(\mathrm{~T}_{\mathrm{w}}-\mathrm{T}_{\mathrm{m}}=20^{\circ} \mathrm{C}\right)$ and $33 \%$ shorter for Case $3\left(\mathrm{~T}_{\mathrm{w}}-\mathrm{T}_{\mathrm{m}}=25^{\circ} \mathrm{C}\right)$ when compared to the corresponding value of case $8\left(\mathrm{~T}_{\mathrm{w}}-\mathrm{T}_{\mathrm{m}}=15^{\circ} \mathrm{C}\right)$. The influence of the outer wall temperature on the radiant heat transfer rate at the shell inner wall is shown in Figure 12. During the time period, $0 \leq \mathrm{t} \leq 4.0 \mathrm{~min}$, the radiation heat transfer rate increases with an increase of the outer wall temperature. However, after this interval all the curves cross each other and an inverse trend is observed. The thermal equilibrium is reached sooner in the case with the high wall and PCM melting temperature difference (higher Stefan and Grashof numbers). The variation of the domain center point temperature as a function of time, for different scattering coefficients is presented in Figure 13. No significant variation within the curves is observed. Attention should be paid to the fact that any change in the driving temperature difference of the system will generate a variation in the Grashof, Stefan and Planck numbers. However, since the temperature changes are relatively small (close to the PCM melting temperature) the Planck number variations are also small.

The predicted melt fraction for all the analyzed study cases has been plotted as a function of the controlling parameters and is shown in Figure 14. A dimensionless group that includes the Fourier, Grashof, Stefan and Planck numbers as well as the absorption optical thickness $\left(\tau_{a}=\kappa_{a} R_{i}\right)$ and single scattering albedo has been created and used as the abscissa in the graph. The following equation is proposed to be used to correlate the melt fraction of all the analyzed cases:

$M F=1.07 \operatorname{erf}\left[0.35\left(F_{o S t e}^{a} \operatorname{Gr}_{R}^{b} P l^{c} \tau_{a}^{d} \omega^{e}\right)^{1.08}\right]$

It was found that, for the exponents, $a=0.5, b=0.25, c=0.01, d=0.015$ and $e=0.025$, all the data merged together in a single line. A dimensionless group that includes the contribution of thermal radiation at the inner wall of the shell has been defined as:

$$
\frac{N u S t e^{a}}{G r_{R}^{b} P l^{c} \tau_{a}^{d} \omega^{e}}
$$


The above quantity includes the modified Nusselt number which has been calculated based on the predicted total heat transfer rate at the inner wall. The aforementioned group has been plotted, for each study case, as a function of the same dimensionless number used in Eqn. [8] and it is also shown in Figure 14. All the results practically merge into a single curve for the exponents: $a=0.5, b=0.25, c=0.01$, $d=0.015$ and $e=0.025$. The following piecewise correlation for the Nusselt number dimensionless group is proposed:

364

$\frac{N u S t e^{a}}{G r_{R}^{b} P l^{c} \tau_{a}^{d} \omega^{e}}=\left\{\begin{array}{cc}1.472-11.89 \xi & 0.04 \leq \xi<0.08 \\ 0.5 \exp \left(-2.5 \xi^{2.4}\right)+\sin (0.07+0.55 \xi), & 0.08 \leq \xi<0.52 \\ 0.753-0.205 \xi-0.0012 \xi^{2}, & 0.52 \leq \xi \leq 3.5\end{array}\right\}$

365

366

367

368

369

370

371

372

373

374

375

376

377

378

379

380

381

382

383

384

385

386

where: $\xi=$ FoSte $^{a} G r_{R}^{b} P l^{c} \tau_{a}^{d} \omega^{e}$

The range of validity of the above correlations can be defined as: $0.038<$ Ste $<0.063,9.9 \times 10^{5}<$ $G r_{R}<5.7 \times 10^{6}, 0.03<P l<0.25,0.5 \leq \tau_{a} \leq 6.0$, and $0.6 \leq \omega \leq 0.9$. It may be noted that this correlation will be quite useful in designing thermal energy storage systems with $\mathrm{NaCl}$ as the phase change material. In order to quantify the contribution of the participating thermal radiation during the melting process, an enhancement factor $(\epsilon)$ has been defined as the ratio between the total and the combined conduction and convection heat transfer rates. This quantity may be used to take into consideration the influence of thermal radiation in the thermal modeling of storage tanks containing encapsulated PCMs where the effective thermal conductivity method is commonly used to estimate the energy transfer process between the heat transfer fluid and the PCM capsule. Figure 14 shows the enhancement factor for study case 3 .

\section{Summary and Conclusions}

A comprehensive analysis of the energy transport due to the simultaneous contribution of convection, radiation and conduction during the melting process of a high temperature phase change material encapsulated in a spherical shell has been presented. The discrete ordinate method was used to solve the equation of radiative transfer and the finite volume scheme was used to solve the equations for mass, momentum and energy conservation. Based on the numerical analysis of the governing equations, the results may be summarized as: 
- The contribution of the radiant energy exchange at the inner shell wall is one order of magnitude smaller than the convective transport process.

- For a fixed capsule size and temperature difference $\Delta T$, the total heat transfer rate at the inner surface of the shell increases by increasing the medium optical thickness. It was shown that, the presence of thermal radiation reduces the melting time by $10 \%$ when compared to the study case with no radiation. However, a significant contribution of radiant heat transfer was found during the solid phase heating process, even for small optical thicknesses. The time to reach $800.2^{\circ} \mathrm{C}$ at the center point is $78.6 \%$ faster in case $3\left(\kappa_{a}=100 m^{-1}\right)$ as compared to the study case with no radiation. No further improvements on the output parameters were obtained when the absorption coefficient varied from 100 to $200 \mathrm{~m}^{-1}$.

- It should be pointed out that the analyzed PCM has a relatively low viscosity value which enhances the natural convection heat transfer. Further analysis should be directed to PCMs with higher viscosities where the viscous dissipation term cannot be neglected.

- For a fixed absorption coefficient and temperature difference $\Delta T$, the increase of the capsule size significantly increases the melting time and radiant heat transfer at the inner surface of the shell. The melting time for the capsule with $R_{i}=0.030 \mathrm{~m}$ is $27.2 \%$ and $64.5 \%$ higher compared to that for the shells with $R_{i}=0.025 \mathrm{~m}$ and $R_{i}=0.020 \mathrm{~m}$ respectively.

- The increase of the outer wall temperature significantly enhances the melting process. The melting time was $20 \%$ shorter for Case $7\left(\mathrm{~T}_{\mathrm{w}}-\mathrm{T}_{\mathrm{m}}=20^{\circ} \mathrm{C}\right)$ and $33 \%$ shorter for Case $3\left(\mathrm{~T}_{\mathrm{w}^{-}}\right.$ $\left.\mathrm{T}_{\mathrm{m}}=25^{\circ} \mathrm{C}\right)$ compared to the corresponding value of case $8\left(\mathrm{~T}_{\mathrm{w}}-\mathrm{T}_{\mathrm{m}}=15^{\circ} \mathrm{C}\right)$.

- Expressions for the melt fraction and modified Nusselt number as a function of the controlling parameters of the process have been developed for latent heat thermal energy storage contained in a packed bed heat exchanger.

\section{Acknowledgements}

The work presented herewith has been funded by the U.S. Department of Energy through the Advanced Research Projects Agency-Energy (ARPA-E) under award number: DE-AR0000179. The authors also would like to acknowledge the use of the services provided by Research Computing at the University of South Florida. 


\section{References}

[1] A. L. Avila-Marin, J. Fernandez-Reche and F. M. Tellez, "Evaluation of the potential of central receiver solar power plants: Configuration, optimization and trends," Applied Energy, vol. 112, pp. 274-288, 2013.

[2] O. Behar, A. Khellaf and K. Mohammedi, "A review of studies on central receiver solar thermal power plants," Renewable and sustainable energy reviews, vol. 23, pp. 12-39, 2013.

[3] U.S. Department of Energy, Energy Efficiency and Renewable Energy, "Sunshot VisionStudy," U.S. DOE, Washington, DC, Report No. DOE/GO-102012-3037, pp. 97-124, 2012.

[4] G. J. Kolb, C. K. Ho, T. R. Mancini and J. A. Gary, "Power tower technology roadmap and cost reduction plan," Sandia National Laboratories, Livermore, CA, Technical Report No. SAND20112419, 2011.

[5] J. Stekli, L. Irwin and R. Pitchumani, "Technical challenges and opportunities for concentrating solar power with thermal energy storage," Journal of Thermal Science and Engineering Applications, vol. 5, p. 021011, 2013.

[6] K. Nithyanandam and R. Pitchumani, "Cost and performance analysis of concentrating solar power systems with integrated latent thermal energy storage," Energy, vol. 64, pp. 793-810, 2014.

[7] J. E. Pacheco, S. K. Showalter and W. J. Kolb, "Development of a molten-salt thermocline thermal storage system for parabolic trough plants," Journal of Solar Energy Engineering, vol. 124, pp. 153$159,2002$.

[8] Electric Power Research Institute (EPRI), "Solar thermocline storage systems: Preliminary Design Study," Electric Power Research Institute, Palo Alto, CA, Report No. 1019581, 2010.

[9] Z. Yang and S. V. Garimella, "Molten-salt thermal energy storage in thermoclines under different environmental boundary conditions," Applied Energy, vol. 87, pp. 3322-3329, 2010.

[10] P. Konakov, "On the regularities of composite heat transfer," International Journal of Heat and Mass Transfer, vol. 2, pp. 136-149, 1961.

[11] E. Sparrow, C. Usiskin and H. Hubbard, "Radiation heat transfer in a spherical enclosure containing a participating, heat-generating gas," Journal of Heat Transfer, vol. 83, pp. 199-206, 1961.

[12] E. A. Dennar and M. Sibulkin, "An Evaluation of the differential approximation for spherically symmetric radiative transfer," Journal of Heat Transfer, vol. 91, pp. 73-76, 1969. 
[13] Y. Chou and C. Tien, "A modified moment method for radiative transfer in non-planar systems," Journal of Quantitative Spectroscopy and Radiative Transfer, vol. 8, pp. 919-933, 1968.

[14] T. W. Tong and P. S. Swathi, "Radiative heat transfer in emitting-absorbing-scattering spherical media," Journal of Thermophysics and Heat Transfer, vol. 1, pp. 162-170, 1987.

[15] I. L. Ryhming, "Radiative transfer between two concentric spheres separated by an absorbing and emitting gas," International Journal of Heat and Mass Transfer, vol. 9, pp. 315-324, 1966.

[16] R. Viskanta and A. Crosbie, "Radiative transfer through a spherical shell of an absorbing-emitting gray medium," Journal of Quantitative Spectroscopy and Radiative Transfer, vol. 7, pp. 871-889, 1967.

[17] G. Jia, Y. Yener and J. Cipolla Jr, "Radiation between two concentric spheres separated by a participating medium," Journal of Quantitative Spectroscopy and Radiative Transfer, vol. 46, pp. 1119, 1991.

[18] R. Viskanta and R. Merriam, "Heat transfer by combined conduction and radiation between concentric spheres separated by radiating medium," Journal of Heat Transfer, vol. 90, pp. 248-256, 1968.

[19] H.-S. Chu and L.-C. Weng, "Transient combined conduction and radiation in anisotropically scattering spherical media," Journal of thermophysics and heat transfer, vol. 6, pp. 553-556, 1992.

[20] R. Viskanta and P. Lall, "Transient cooling of a spherical mass of high-temperature gas by thermal radiation," Journal of Applied Mechanics, vol. 32, pp. 740-746, 1965.

[21] S. Thynell and M. Özisik, "Radiation transfer in an isotropically scattering homogeneous solid sphere," Journal of Quantitative Spectroscopy and Radiative Transfer, vol. 33, pp. 319-330, 1985.

[22] J. Tsai and M. Özisik, "Transient, combined conduction and radiation in an absorbing, emitting, and isotropically scattering solid sphere," Journal of Quantitative Spectroscopy and Radiative Transfer, vol. 38, pp. 243-251, 1987.

[23] C. Siewert and J. Thomas, "On coupled conductive-radiative heat transfer problems in a cylinder," Journal of Quantitative Spectroscopy and Radiative Transfer, vol. 48, pp. 227-236, 1992.

[24] Y. Bayazitoglu and P. Suryanarayana, "Transient radiative heat transfer from a sphere surrounded by a participating medium," Journal of Heat Transfer, vol. 111, pp. 713-718, 1989.

[25] S. Thynell, "Interaction of conduction and radiation in anisotropically scattering, spherical media," Journal of thermophysics and heat transfer, vol. 4, pp. 299-304, 1990.

[26] M. Abrams and R. Viskanta, "The effects of radiative heat transfer upon the melting and solidification of semitransparent crystals," Journal of Heat Transfer, vol. 96, pp. 184-190, 1974. 
[27] R. Raj, A. Prasad, P. R. Parida and S. C. Mishra, "Analysis of solidification of a semitransparent planar layer using the lattice Boltzmann method and the discrete transfer method," Numerical Heat Transfer, Part A: Applications, vol. 49, pp. 279-299, 2006.

[28] P. Lapka and P. Furmanski, "Numerical modelling of solidification processes of semitransparent materials using the enthalpy and the finite volume methods," Heat and Mass Transfer, vol. 44, pp. 937-957, 2008.

[29] E. Sparrow and J. Broadbent, "Freezing in a vertical tube," Journal of Heat Transfer, vol. 105, pp. 217-225, 1983.

[30] A. R. Archibold, M. M. Rahman, D. Y. Goswami and E. K. Stefanakos, "Numerical solution of heat transfer during solidification of an encapsulated phase change material," in Proceedings of the ASME 2013 International Mechanical Engineering Congress \& Exposition, Nov. 15-21, San Diego, CA, 2013.

[31] J. Miranda Fuentes, K. Johannes, F. Kuznik, M. Cosnier and J. Virgone, "Melting with convection and radiation in a participating phase change material," Applied Energy, vol. 109, pp. 454-461, 2013.

[32] B. Webb and R. Viskanta, "Radiation-induced melting with buoyancy effects in the liquid," Experimental Heat Transfer, vol. 1, pp. 109-126, 1987.

[33] S. Chandrasekhar, Radiative transfer, New York: Dover Publications, 1960.

[34] R. Viskanta and M. Mengüc, "Radiation heat transfer in combustion systems," Progress in Energy and Combustion Science, vol. 13, pp. 97-160, 1987.

[35] W. Fiveland, "Discrete-ordinates solutions of the radiative transport equation for rectangular enclosures," Journal of Heat Transfer, vol. 106, pp. 699-706, 1984.

[36] K. Sen and S. J. Wilson, Radiative transfer in curved media: basic and mathematical methods for radiative transfer and transport problems in participating media of spherical and cylindrical geometry, World Scientific, 1990.

[37] R. Siegel and J. Howell, Thermal radiation heat transfer, Taylor \& Francis, 2002.

[38] S. V. Patankar, Numerical heat transfer and fluid flow, Taylor \& Francis, 1980.

[39] A. R. Archibold, A. Bhardwaj, M. M. Rahman, D. Y. Goswami and E. L. Stefanakos, "Thermal assessment of a latent leat energy storage module using a high temperature phase change material with enhanced radiative properties," in Proceedings of the ASME 2014 International Mechanical Engineering Congress \& Exposition, Nov. 14-20, Montreal, Canada, 2014.

[40] Y. Touloukian and D. DeWitt, "Thermophysical properties of matter-The TPRC data series. Volume 8. Thermal radiative properties-nonmetallic solids.," 1972. 
[41] A. R. Archibold, J. Gonzalez-Aguilar, M. M. Rahman, D. Yogi Goswami, M. Romero and E. K. Stefanakos, "The melting process of storage materials with relatively high phase change temperatures in partially filled spherical shells," Applied Energy, vol. 116, pp. 243-252, 2014.

[42] A. R. Archibold, M. M. Rahman, D. Y. Goswami and E. K. Stefanakos, "Analysis of heat transfer and fluid flow during melting inside a spherical container for thermal energy storage," Applied Thermal Engineering, vol. 64, pp. 396-407, 2014.

[43] M. Chase, "NIST-JANAF Thermochemical Tables, Monograph 9," J. Phys. Chem. Ref. Data, vol. 27, p. 1951, 1998.

[44] M. Smirnov, V. Khokhlov and E. Filatov, "Thermal conductivity of molten alkali halides and their mixtures," Electrochimica acta, vol. 32, pp. 1019-1026, 1987.

[45] G. J. Janz, "Molten salts data as reference standards for density, surface tension, viscosity and electrical conductance: KNO3 and NaCl," J. Phys. Chem. Ref. Data, vol. 9, pp. 791-829, 1980.

[46] H. L. Johnston and D. A. Hutchison, "Density of Sodium Chloride the atomic weight of Fluorine by combination of crystal density and x-ray data," Physical Review, vol. 62, pp. 32-36, 1942. 


\section{Nomenclature}

$\mathrm{c}_{\mathrm{p}} \quad$ specific heat at constant pressure $(\mathrm{J} / \mathrm{kg} \mathrm{K})$

$\Delta T \quad$ temperature difference $\left({ }^{\circ} \mathrm{C}\right)\left(T_{w}-T_{m}\right)$

Fo Fourier number $\left(\kappa / \rho \mathrm{c}_{\mathrm{p}}\right)\left(\mathrm{t} / \mathrm{R}_{\mathrm{i}}{ }^{2}\right)$

g gravitational acceleration $\left(\mathrm{m} / \mathrm{s}^{2}\right)$

$G r_{R} \quad$ Grashof number $\left(g \beta \Delta T R_{i}^{3} \rho^{2} / \eta^{2}\right)$

$\hbar$ heat transfer coefficient $\left(\mathrm{W} / \mathrm{m}^{2} \mathrm{~K}\right)\left(\hbar=q_{t} / A \Delta T\right)$

$h \quad$ sensible enthalpy $(\mathrm{J} / \mathrm{kg})$

L Latent heat of fusion $(\mathrm{J} / \mathrm{kg})$

$\mathrm{n} \quad$ index of refraction

$\mathrm{Nu} \quad$ Nusselt number $\left(\hbar R_{i} / \kappa\right)$

$\mathrm{P} \quad$ pressure $(\mathrm{Pa})$

Pl Planck number $\kappa\left(\kappa_{a}+\sigma_{s}\right) / 4 n \sigma \bar{T}^{3}$

Pr Prandtl number of the fluid $\left(\eta c_{p} / k\right)$

$q_{t} \quad$ total heat transfer rate at the inner shell wall (W)

$\mathrm{R}_{\mathrm{i}} \quad$ inner radius of the capsule $(\mathrm{m})$

$\mathrm{Ra}$ Rayleigh number ( $\mathrm{GrPr})$

Ste Stefan number $\left(c_{p}\left(T_{w}-T_{m}\right) / L\right)$

$\mathrm{t}$ time (s)

$\mathrm{T}$ temperature $\left({ }^{\circ} \mathrm{C}\right)$

$\bar{T} \quad$ reference temperature $\left({ }^{\circ} \mathrm{C}\right)\left(T_{w}+T_{m} / 2\right)$

$v \quad$ velocity $(\mathrm{m} / \mathrm{s})$

\section{Greek Symbols}

$\alpha \quad$ Thermal diffusivity $\left(\mathrm{m}^{2} / \mathrm{s}\right)$

$\beta \quad$ thermal expansion coefficient $(1 / \mathrm{K})$

$\delta \quad$ thickness (m)

$\eta \quad$ dynamic viscosity $(\mathrm{kg} / \mathrm{m} \mathrm{s})$

$\kappa \quad$ thermal conductivity $(\mathrm{W} / \mathrm{m} \mathrm{K})$

$\kappa_{a} \quad$ absorption coefficient $\left(\mathrm{m}^{-1}\right)$

$\lambda \quad$ latent heat $(\mathrm{J} / \mathrm{kg})$

$\Theta_{n} \quad$ dimensionless temperature at the center point, $\Theta_{n}=\left(T-T_{o}\right) /\left(T_{w}-T_{o}\right)$

$\rho \quad$ density $\left(\mathrm{kg} / \mathrm{m}^{3}\right)$

$\sigma \quad$ Stefan-Boltzmann constant, $\sigma=5.67 \times 10^{-8} \mathrm{~W} / \mathrm{m}^{2} \mathrm{~K}^{4}$

$\sigma_{s} \quad$ scattering coefficient $\left(\mathrm{m}^{-1}\right)$

$\tau \quad$ optical thickness, $\tau=\left(\kappa_{a}+\sigma_{s}\right) R_{i}$

$\varphi$ dimensionless irradiation

$\xi$ dimensionless stream line value

$\omega \quad$ scattering albedo, $\omega=\sigma_{s} /\left(\kappa_{a}+\sigma_{s}\right)$

\section{Subscripts}

m melting

o initial

$\mathrm{r} \quad$ radial direction

s solid 
w wall

$\theta \quad$ angular direction

427

$428 \quad$ Figure captions

429

430 Figure 1. Schematic representation of the physical domain

431 Figure 1. Influence of the grid size

432 Figure 2 . Effect of the geometric parameter $\left(\boldsymbol{\xi}=\boldsymbol{R}_{\mathbf{1}} / \boldsymbol{R}_{\mathbf{2}}\right)$ on the temperature distribution

433 Figure 3 . The effect of $\kappa_{a}$ on the melt fraction

434 Figure 4 . The effect of $\kappa_{a}$ on the total heat transfer rate at the inner wall

435 Figure 5. The effect of $\kappa_{a}$ on the radiant heat transfer rate at the inner wall

436 Figure 6 . The effect of $\kappa_{a}$ on the center point temperature

437 Figure 7 . The effect of $R_{i}$ on the total heat transfer rate at the inner wall

438 Figure 8 . The effect of $R_{i}$ on the radiant heat transfer rate at the inner wall

439 Figure 9. Predicted evolution of the melting process for study case 5. For each subfigure, (left) incident irradiation contours, (right) PCM solid fraction in gray and PCM liquid streamline contours.

441 Figure 10. The effect of $T_{w}$ on the melt fraction

442 Figure 11. The effect of $T_{w}$ on the radiant heat transfer rate at the inner wall

443 Figure 12. The effect of $\sigma_{s}$ on the center point temperature

444 Figure 13. Melt fraction and Nusselt number as a function of the controlling parameters

445

446

Table captions

447

448 Table 1. PCM Thermo-physical properties

449 Table 2. Dimensionless total and radiative heat fluxes

450 Table 3. Analyzed study cases 


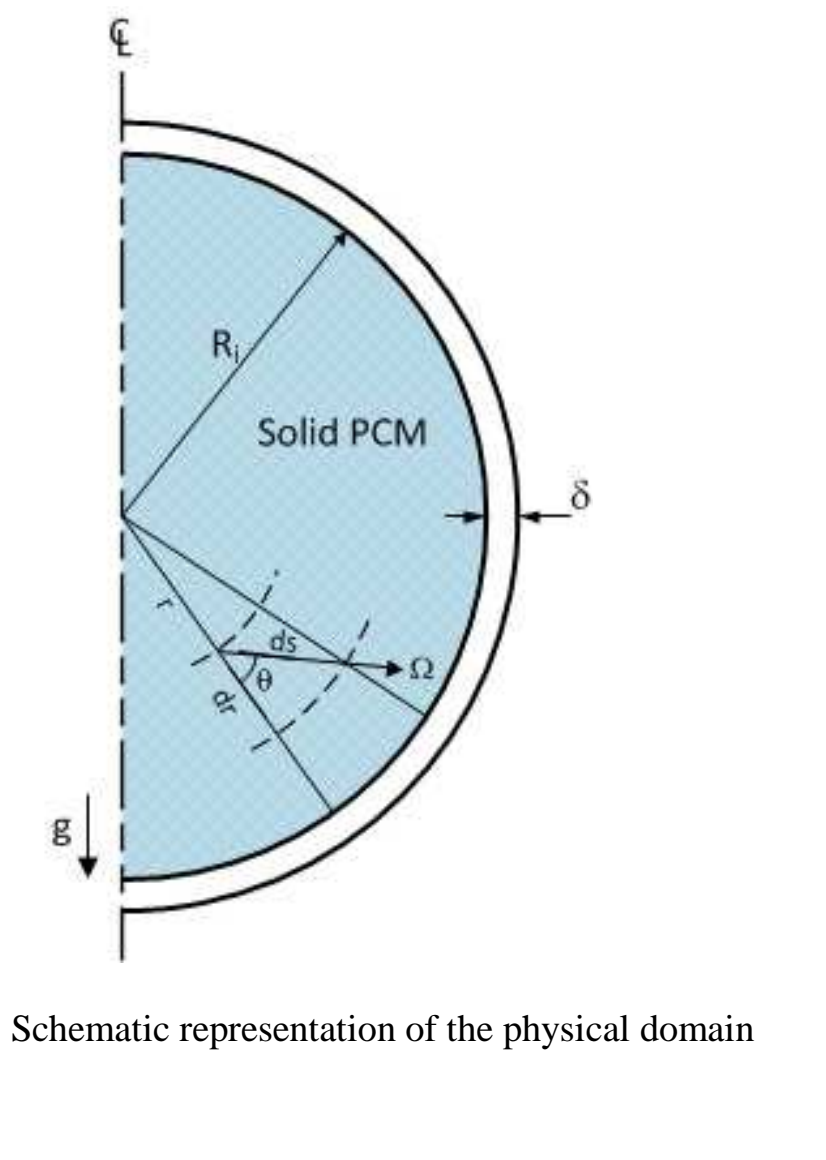

Figure 1. Schematic representation of the physical domain

Figure

.

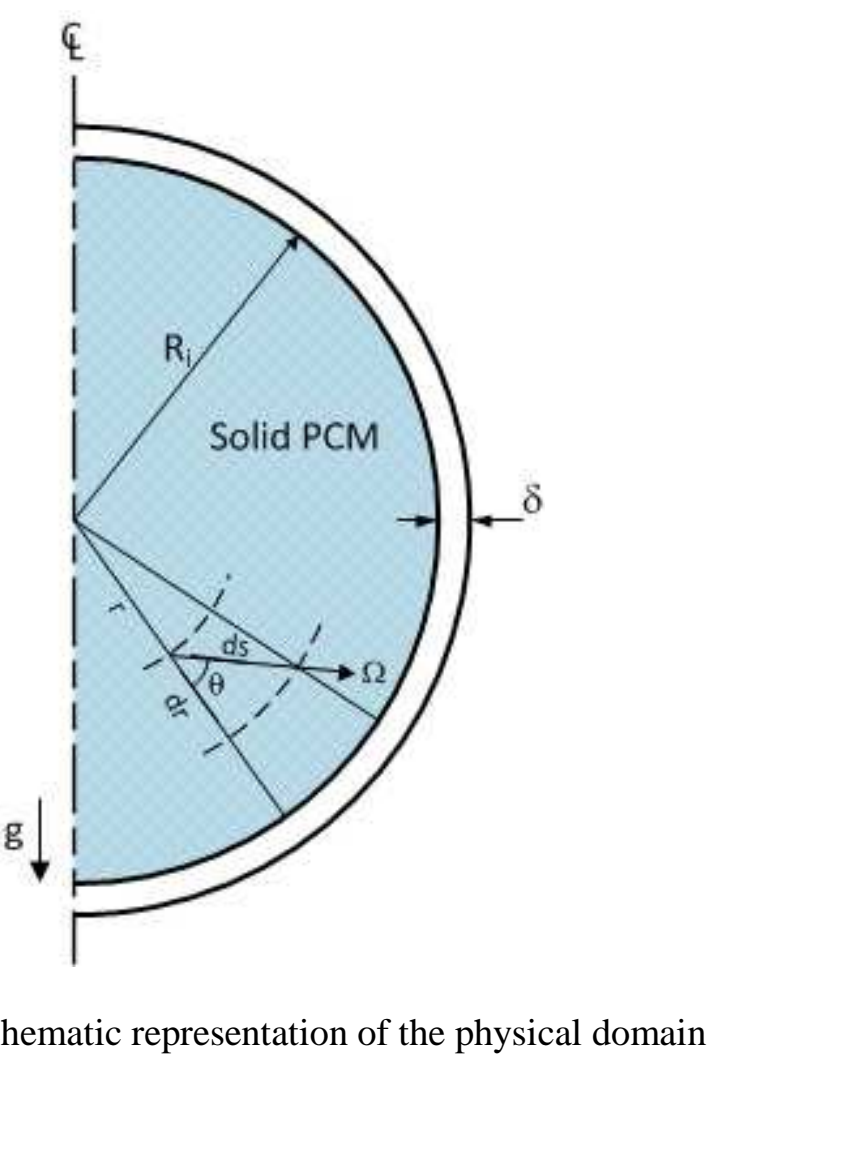

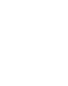

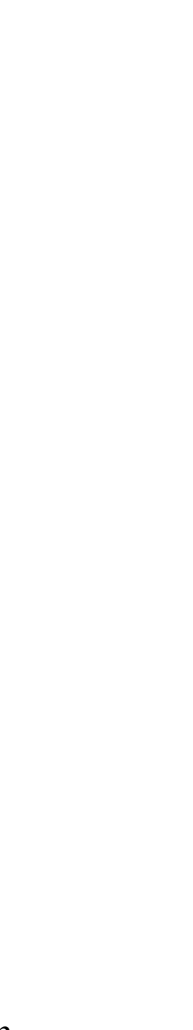

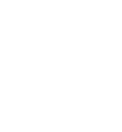

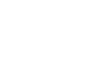




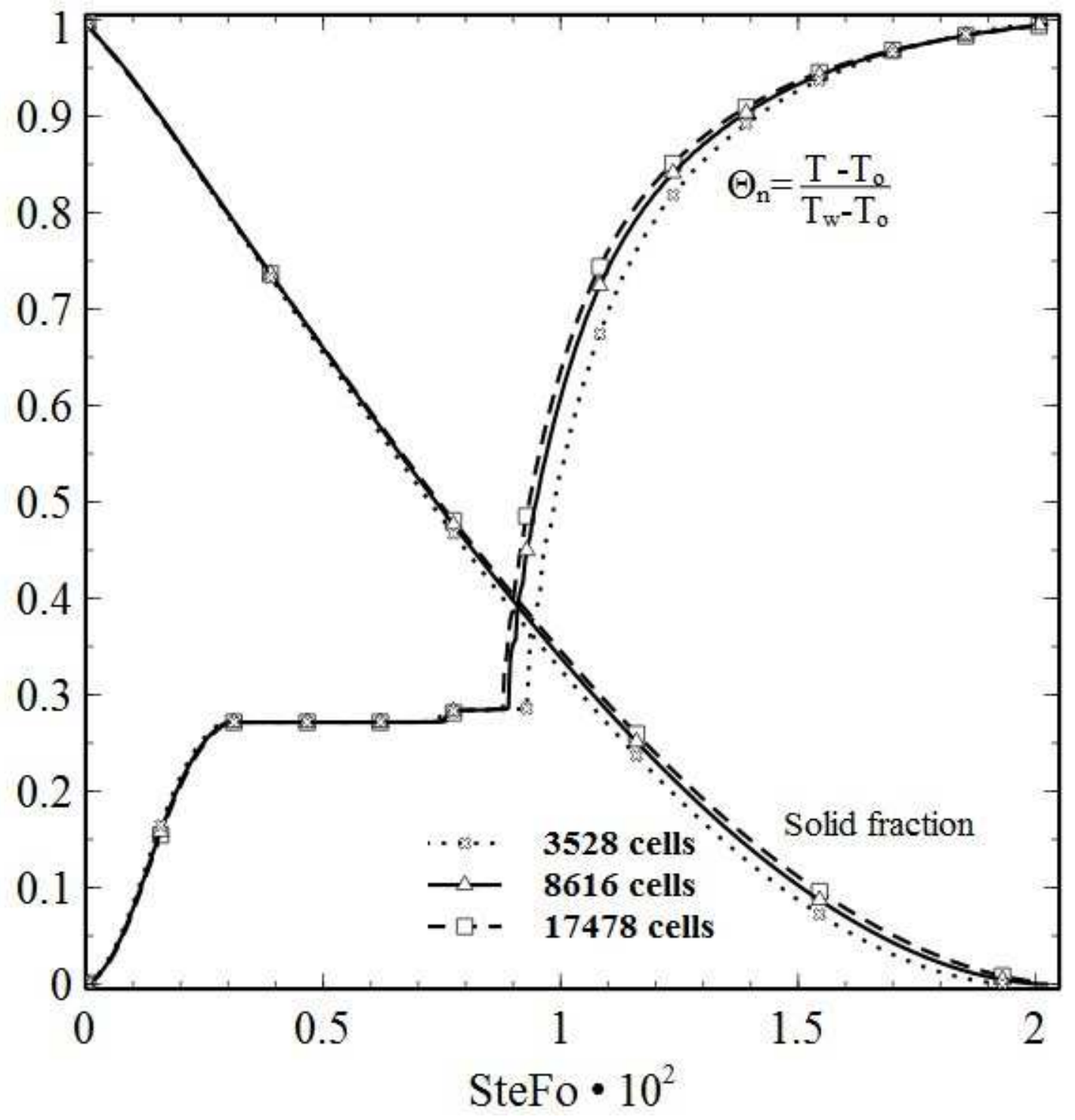

Figure 2 Influence of the grid size 


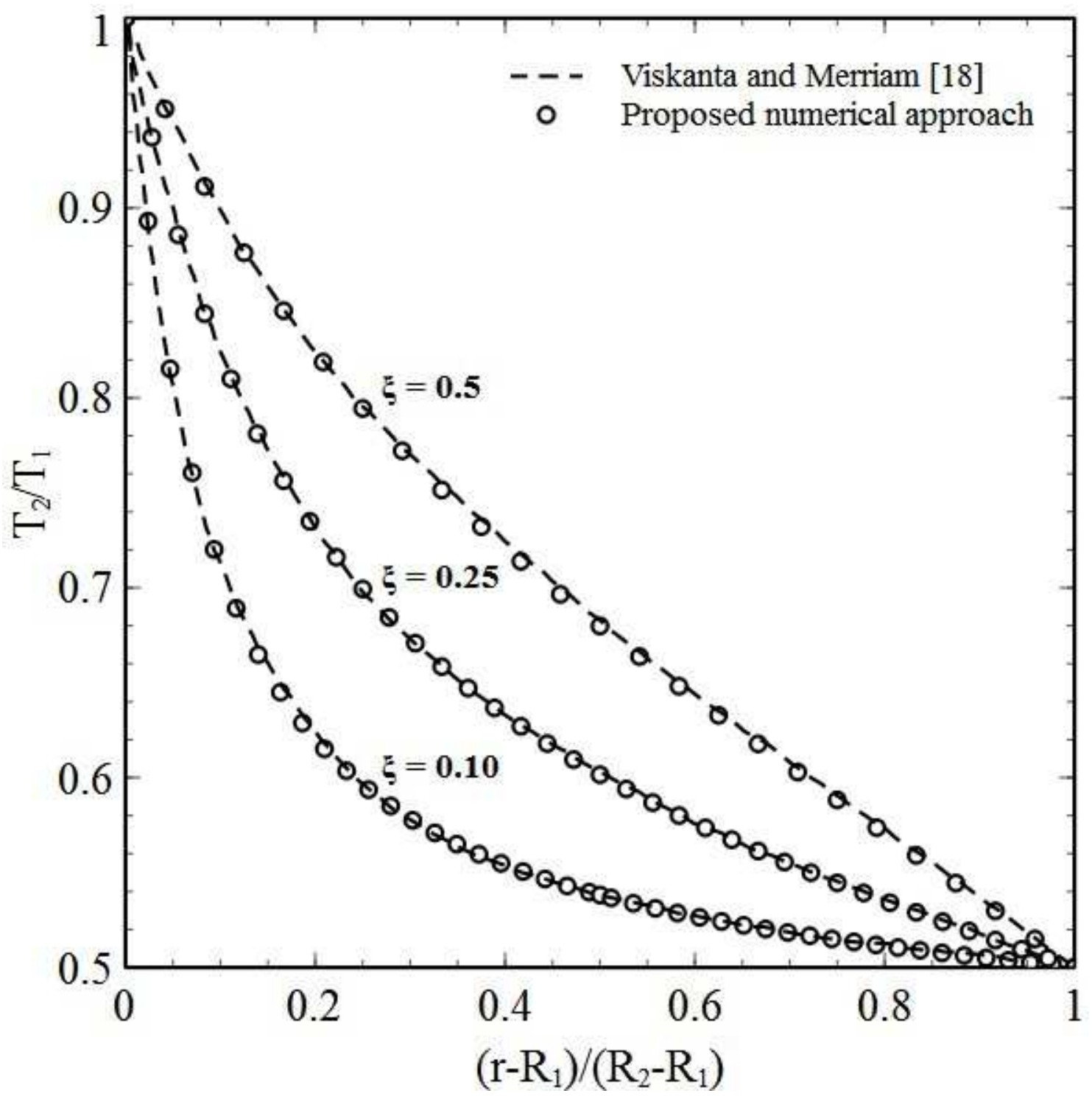

Figure 3 Effect of the geometric parameter $\left(\xi=\boldsymbol{R}_{\mathbf{1}} / \boldsymbol{R}_{\mathbf{2}}\right)$ on the temperature distribution 


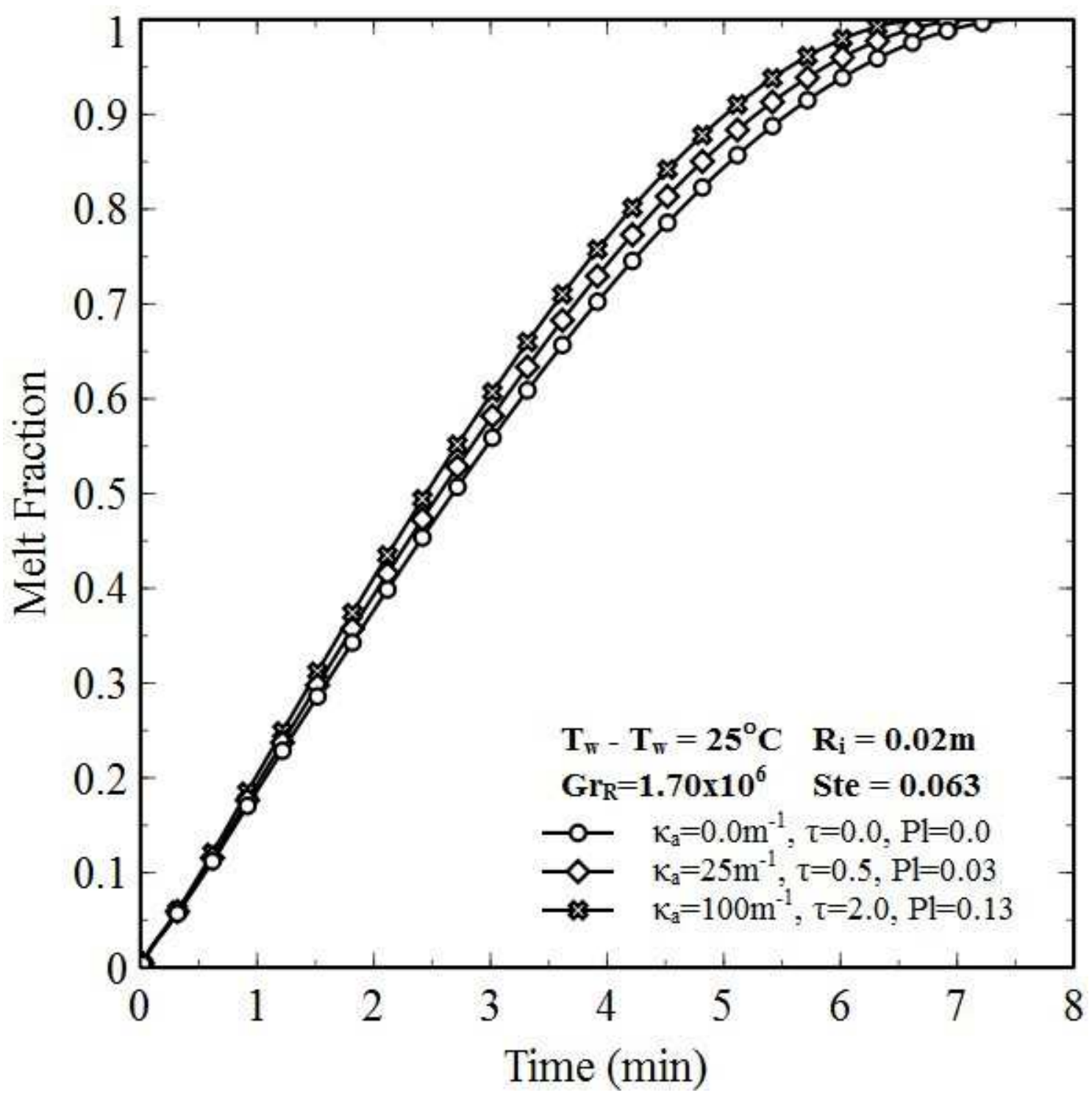

Figure 4 The effect of $\kappa_{a}$ on the melt fraction 


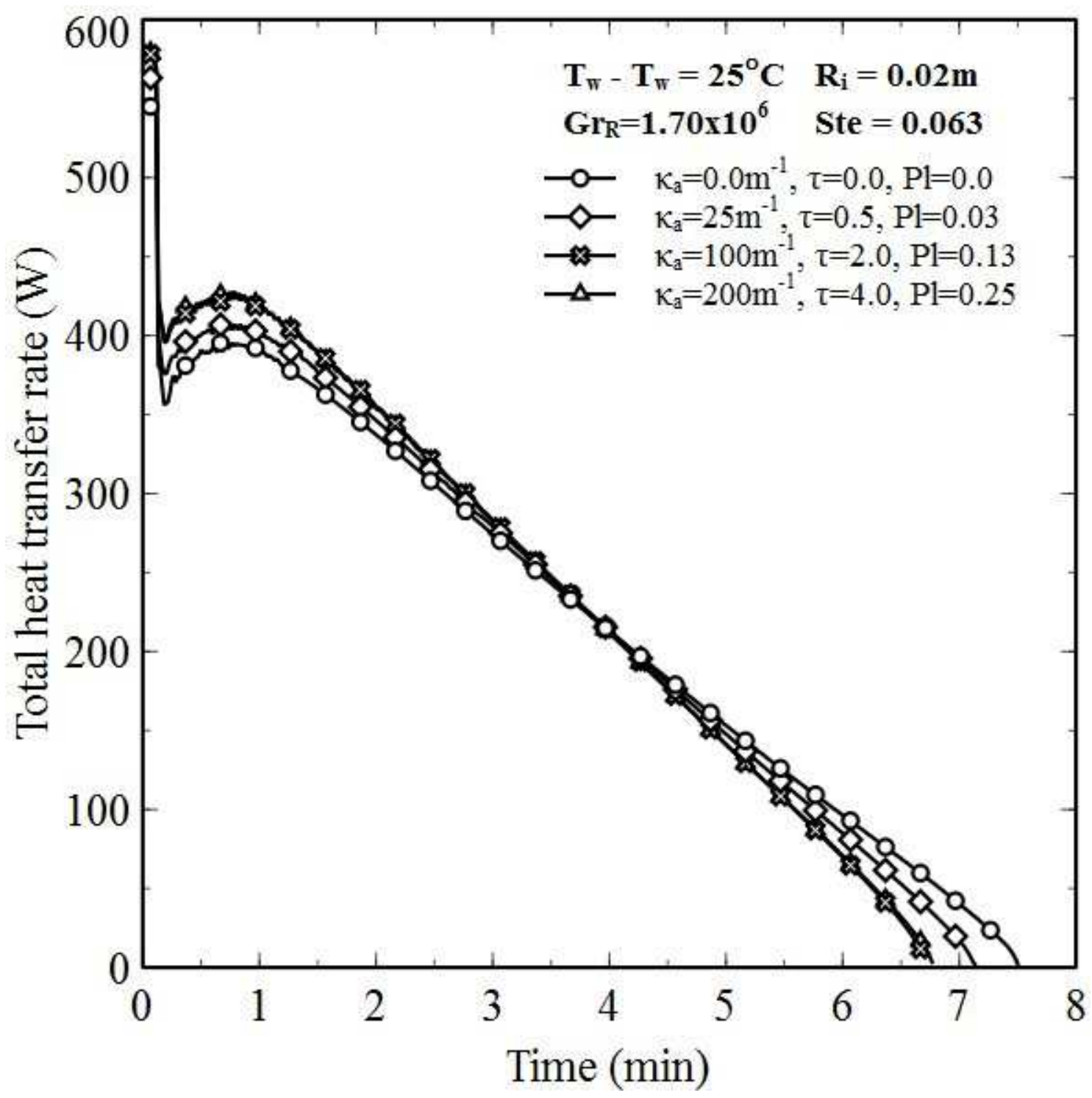

Figure 5 The effect of $\kappa_{a}$ on the total heat transfer rate at the inner wall 


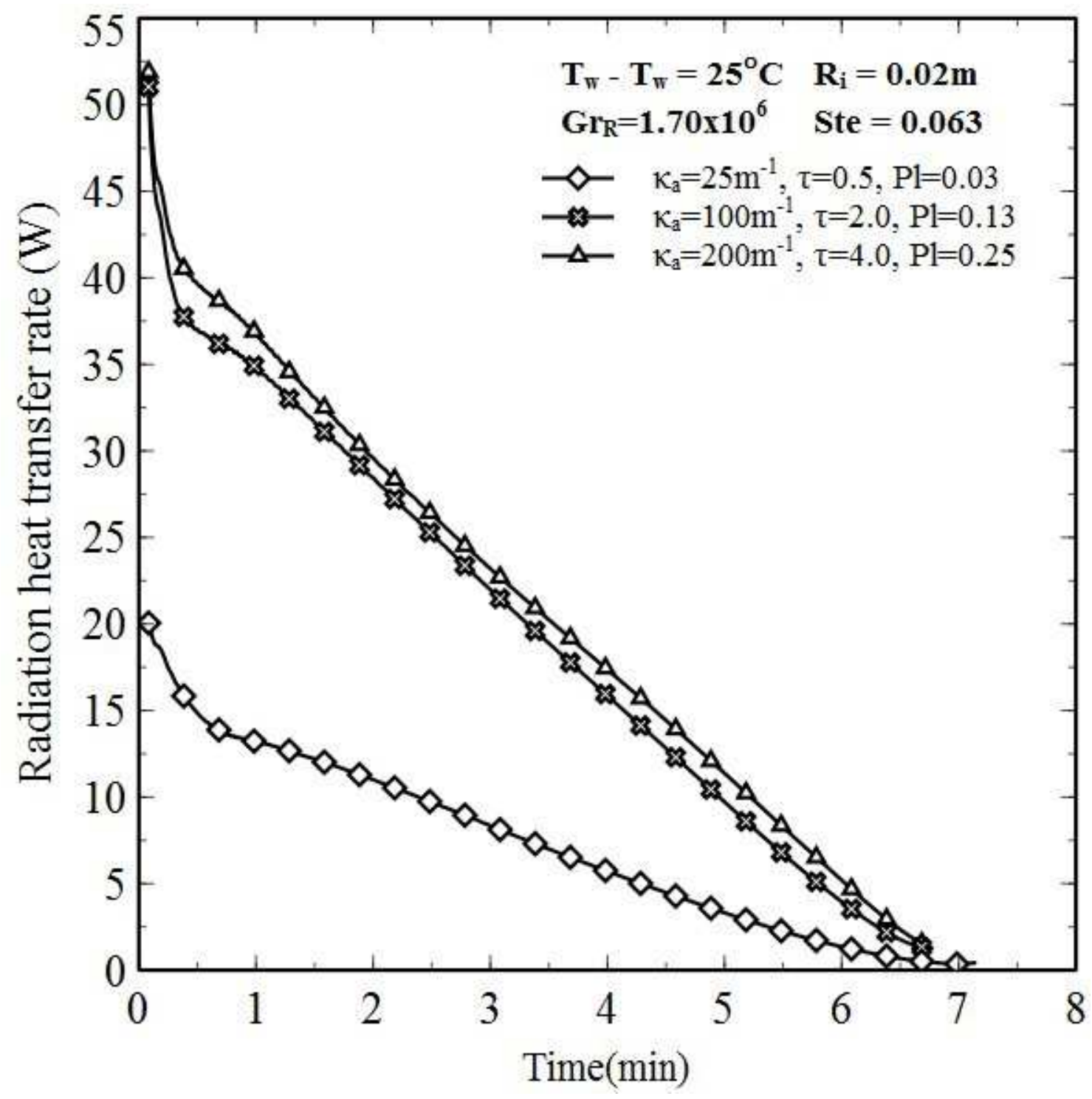

Figure 6 The effect of $\kappa_{a}$ on the radiant heat transfer rate at the inner wall 


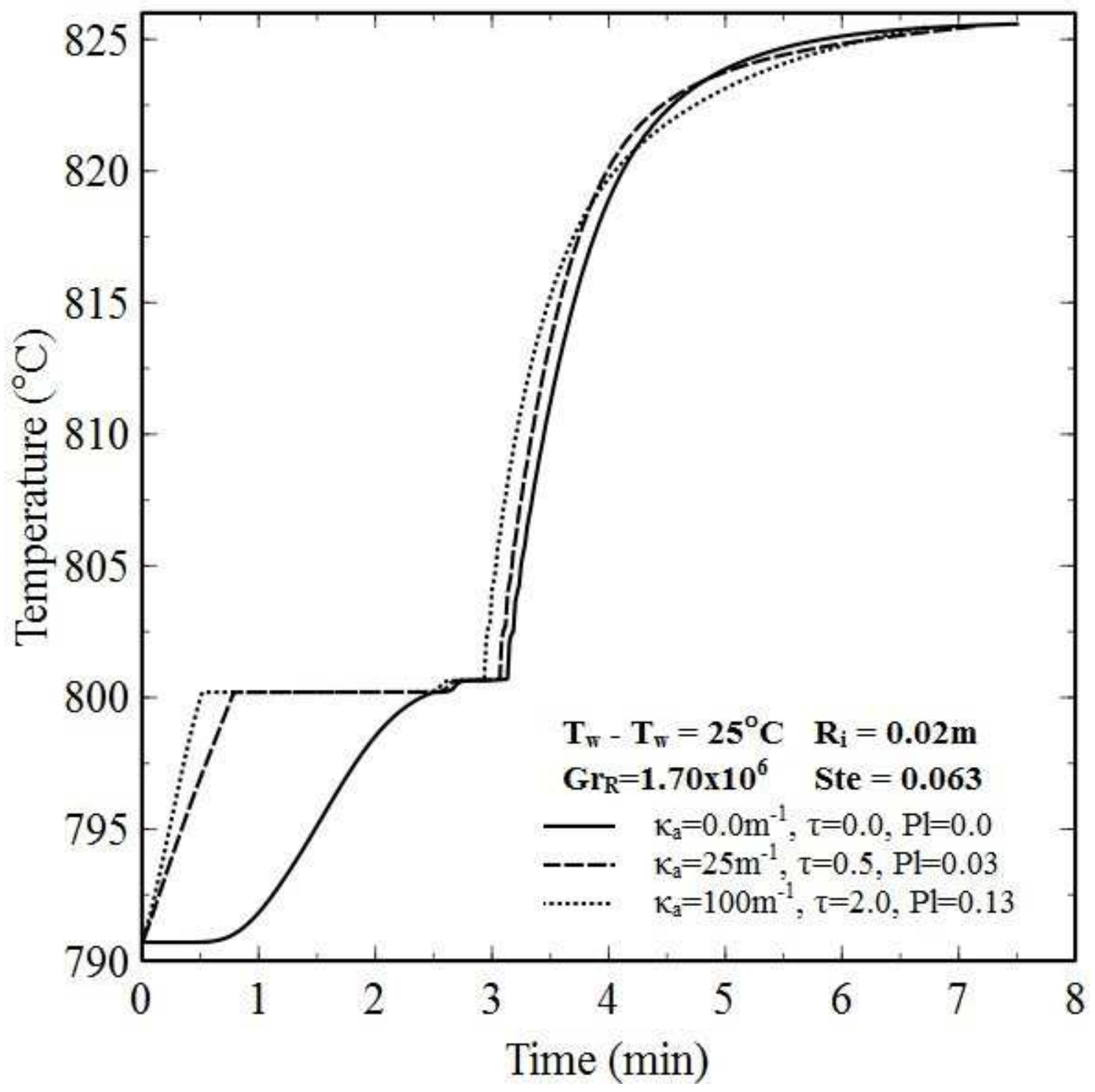

Figure 7 The effect of $\kappa_{a}$ on the center point temperature 


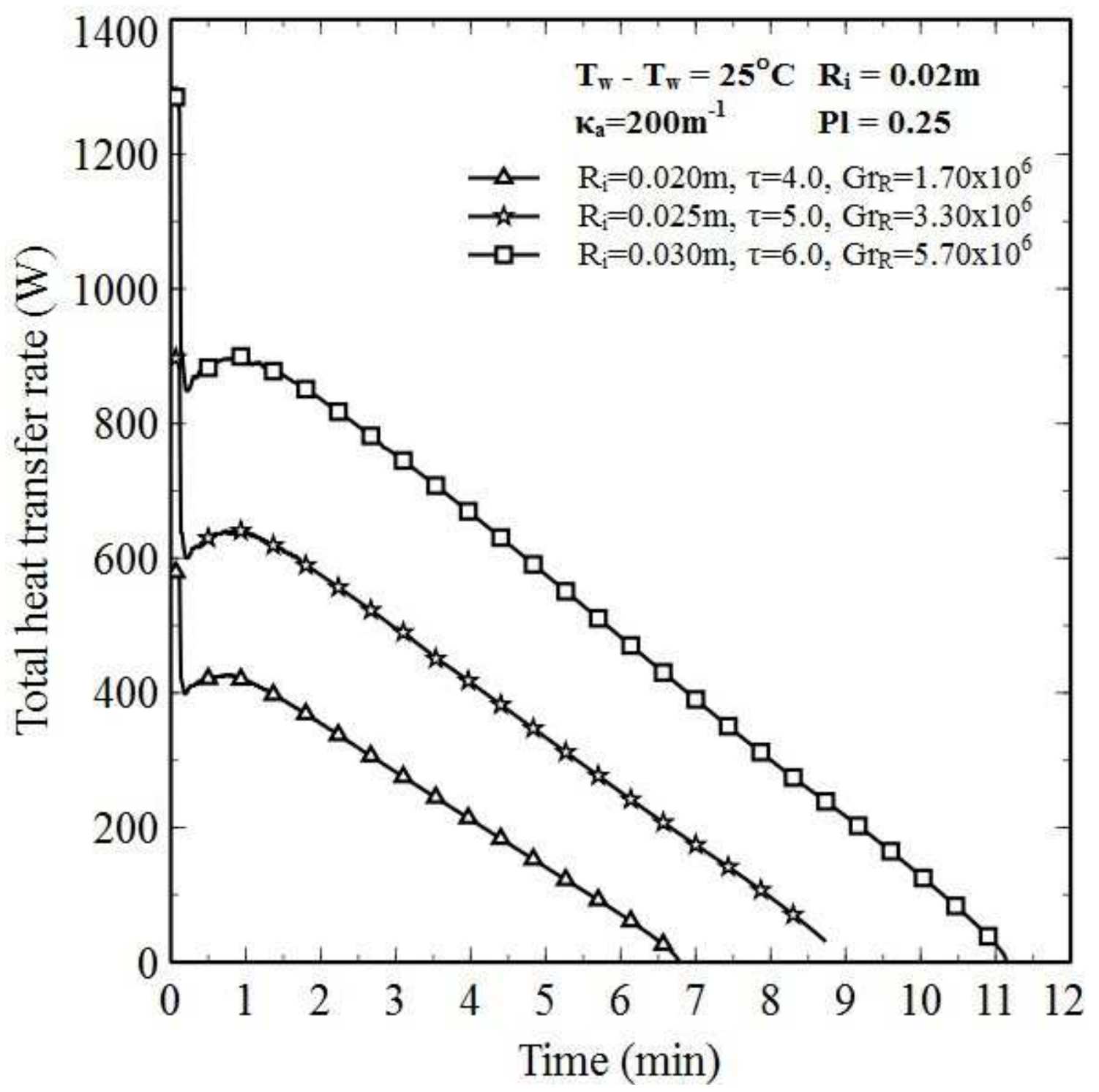

Figure 8 The effect of $R_{i}$ on the total heat transfer rate at the inner wall 


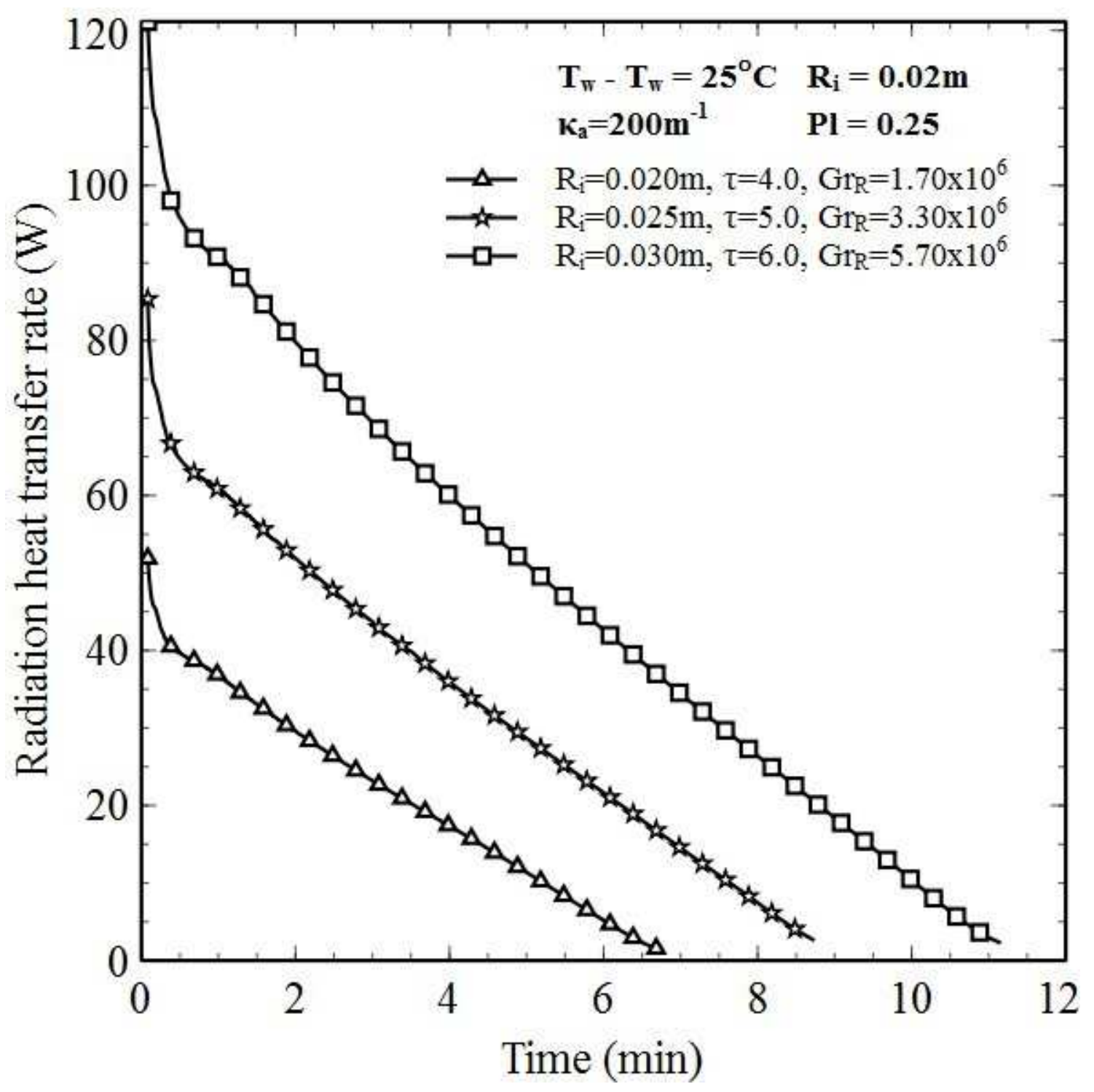

Figure 9 The effect of $R_{i}$ on the radiant heat transfer rate at the inner wall 


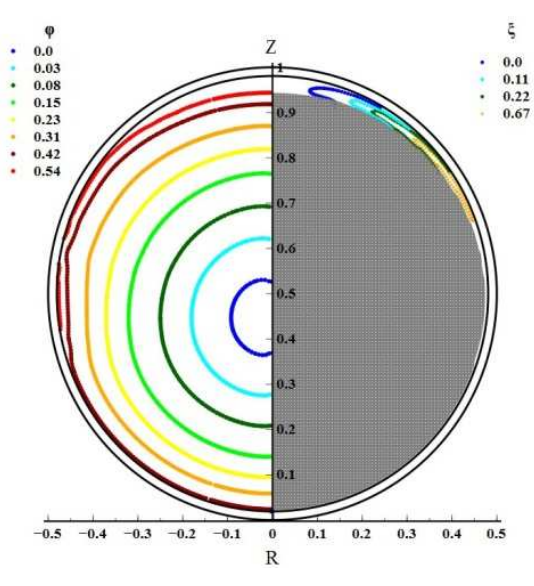

a) Ste $F o=7.70 \times 10^{-4}$

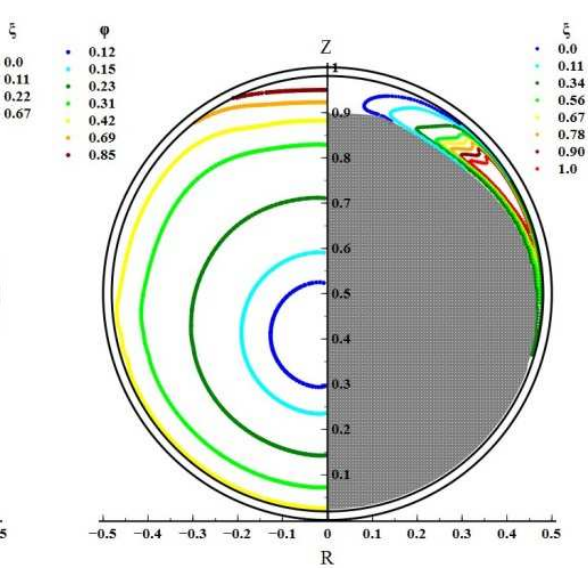

b) Ste $F o=1.54 \times 10^{-3}$

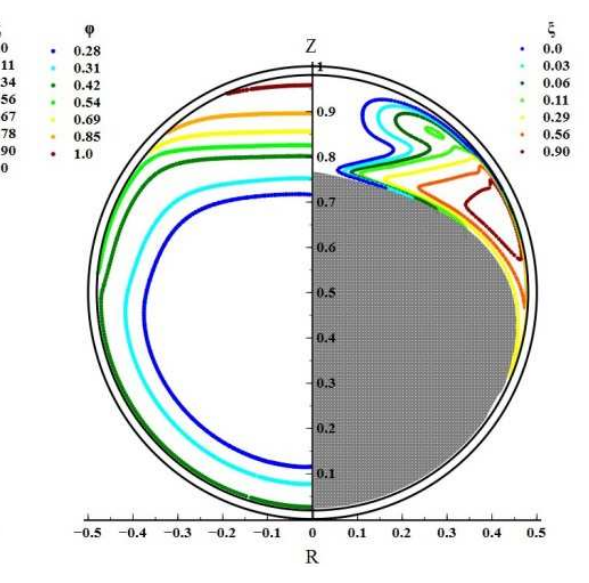

c) Ste $F o=3.08 \times 10^{-3}$

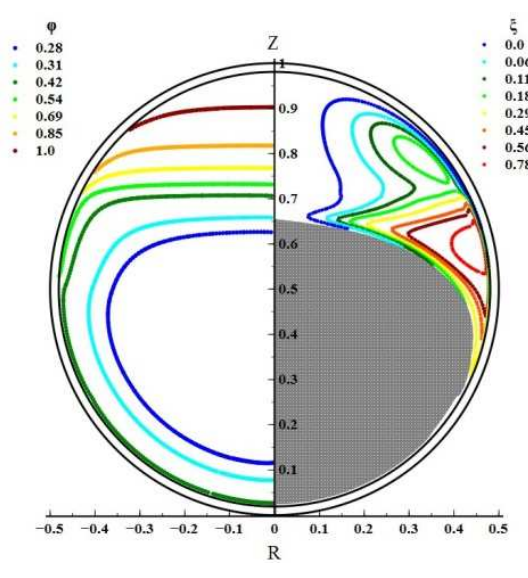

d) Ste $F_{O}=4.62 \times 10^{-3}$

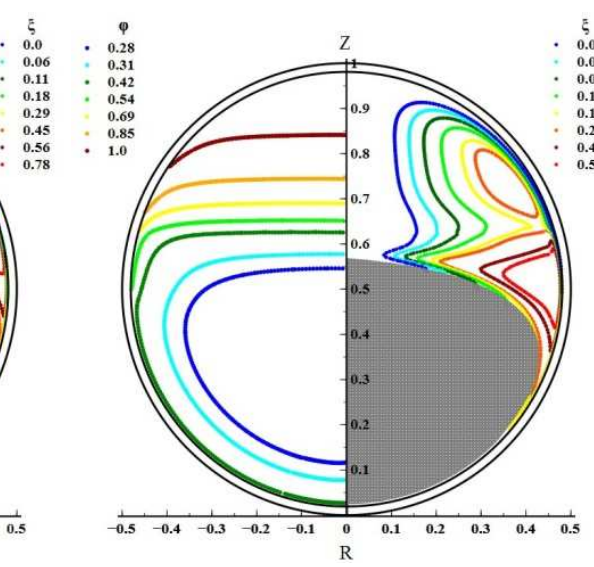

e) Ste $F o=6.16 \times 10^{-3}$

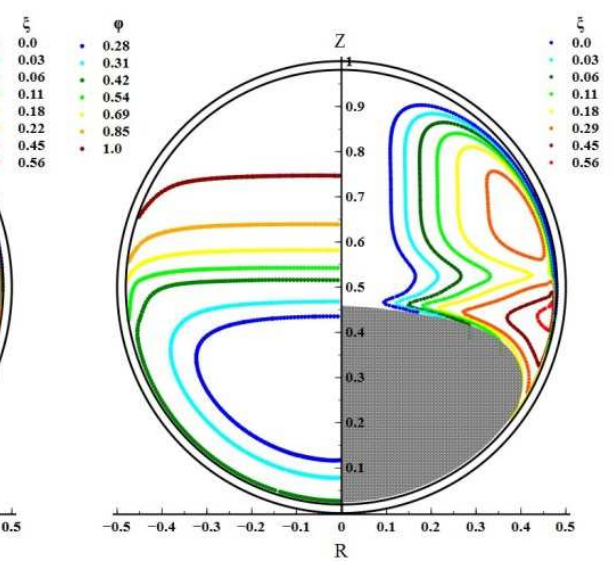

f) Ste $F o=8.47 \times 10^{-3}$

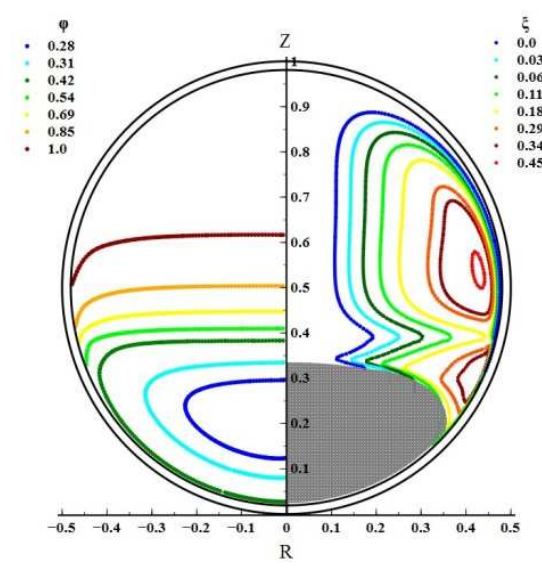

g) Ste $F o=1.15 \times 10^{-2}$

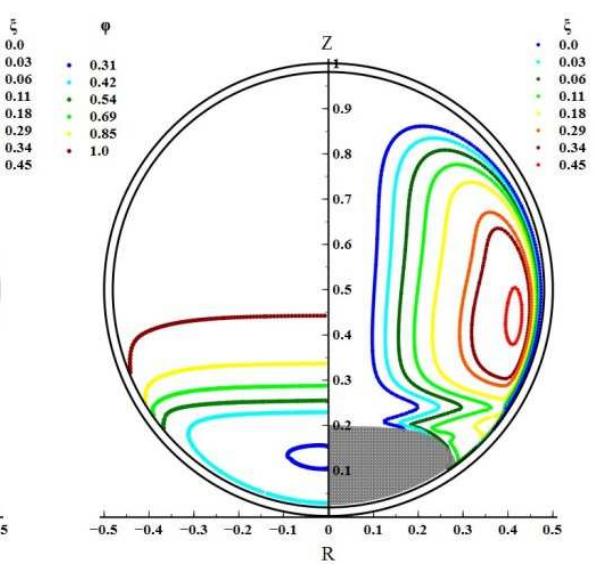

h) Ste $F o=1.54 \times 10^{-2}$

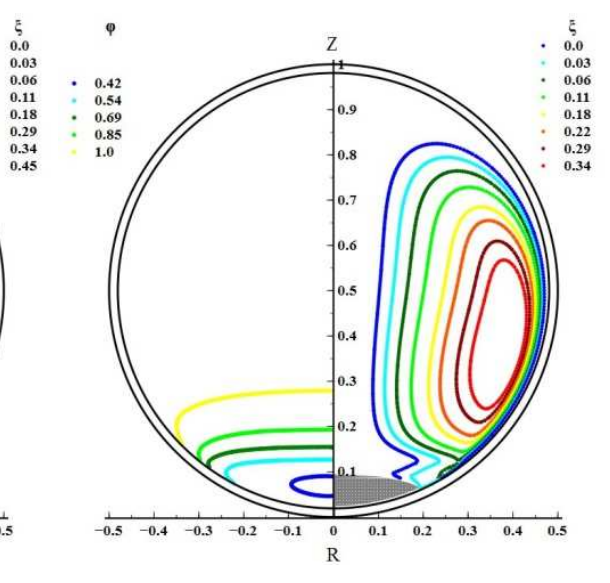

i) Ste $F_{0}=1.85 \times 10^{-2}$

Figure 10 Predicted evolution of the melting process for study case 5. For each subfigure, (left) incident irradiation contours, (right) PCM solid fraction in gray and PCM liquid streamline contours. 


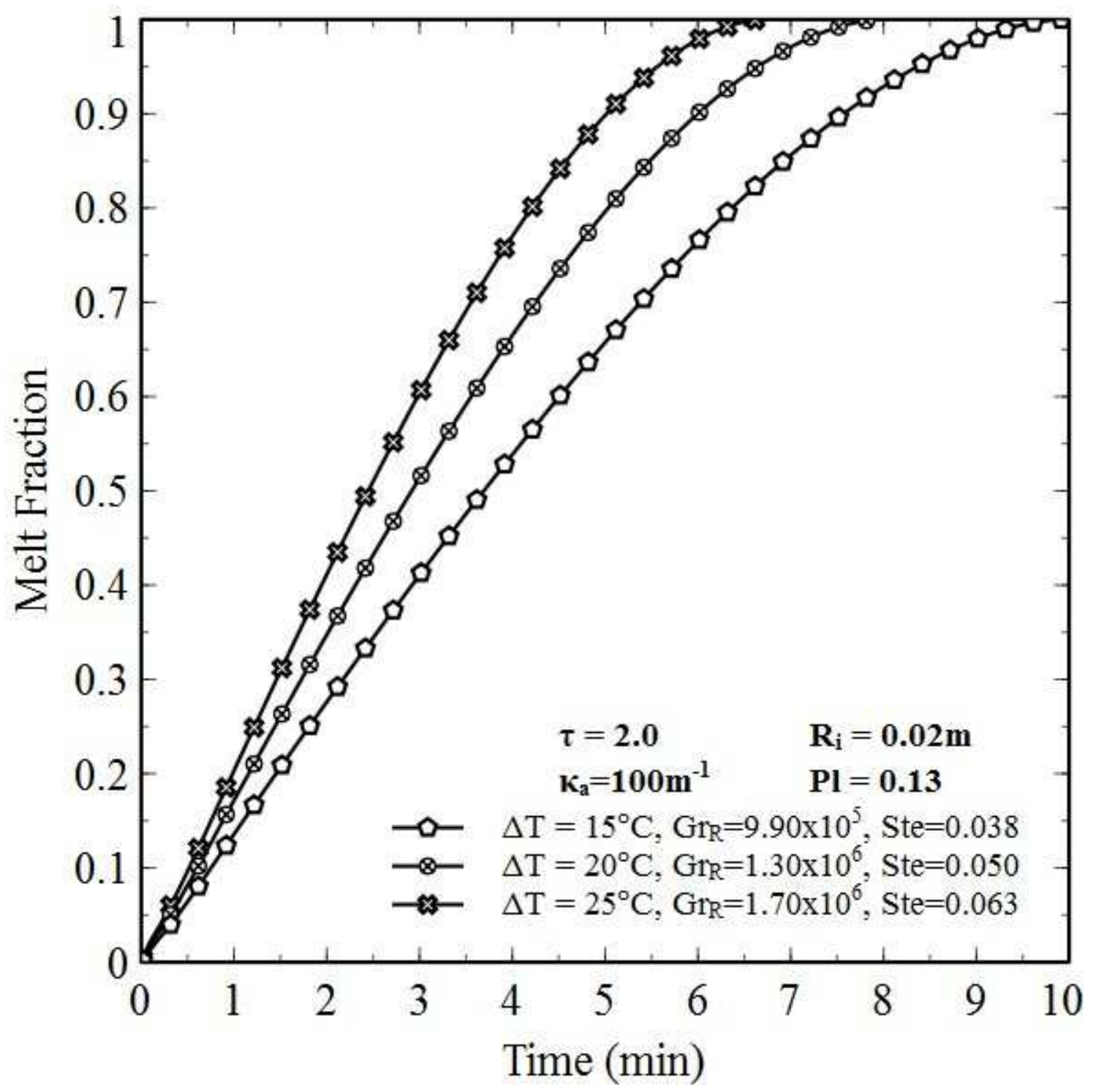

Figure 11 The effect of $T_{w}$ on the melt fraction 


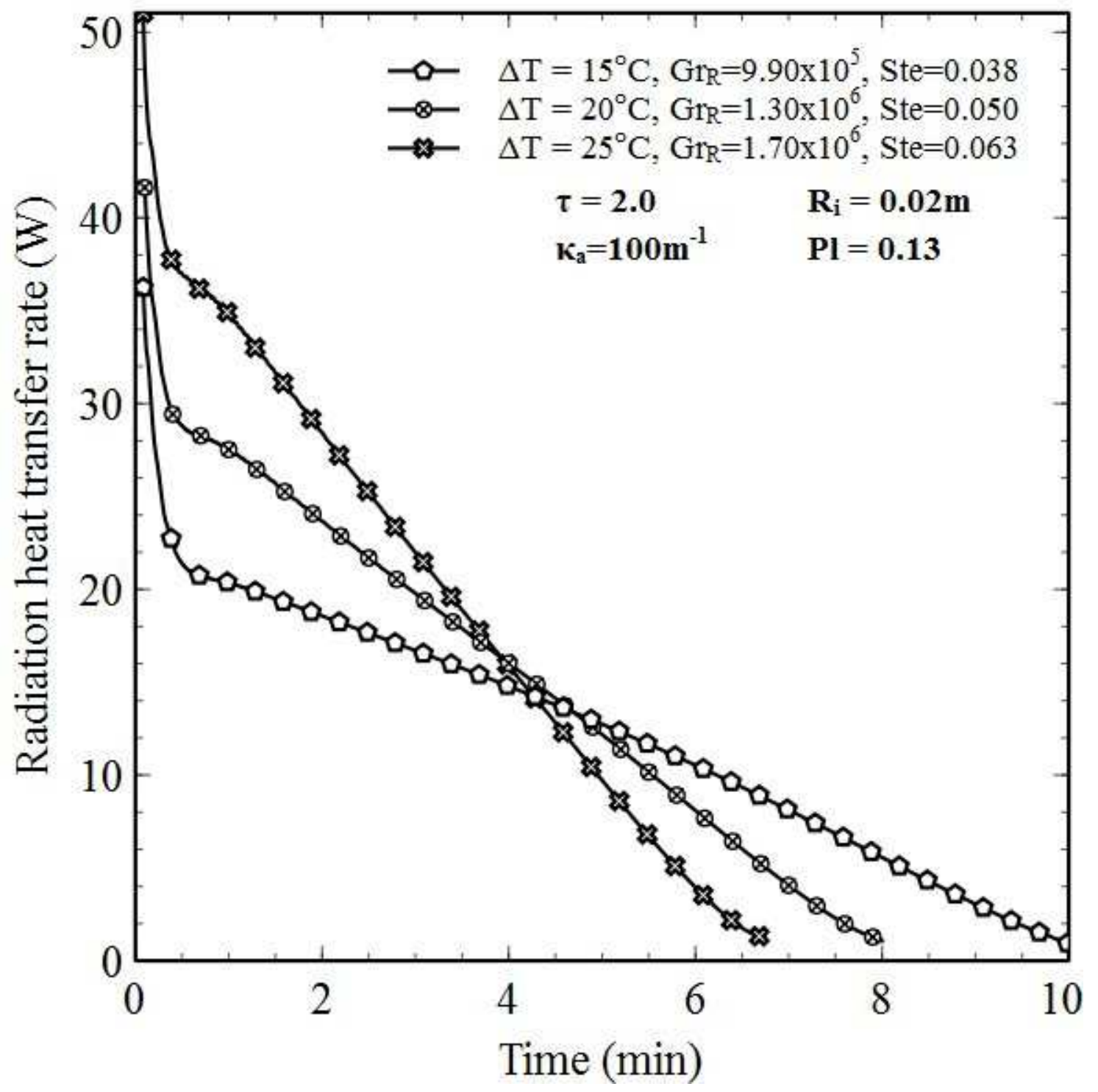

Figure 12 The effect of $T_{w}$ on the radiant heat transfer rate at the inner wall 


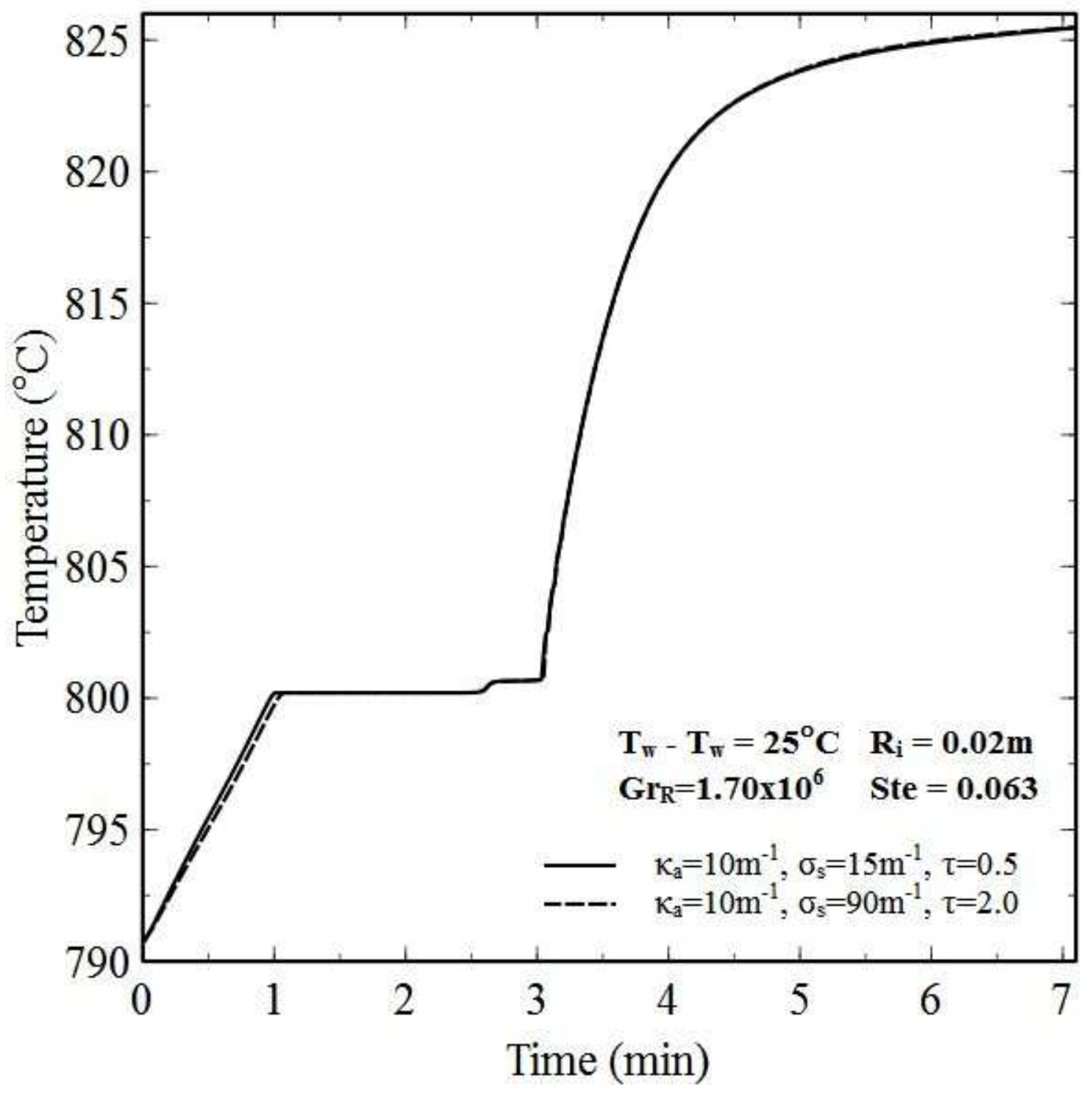

Figure 13 The effect of $\sigma_{s}$ on the center point temperature 


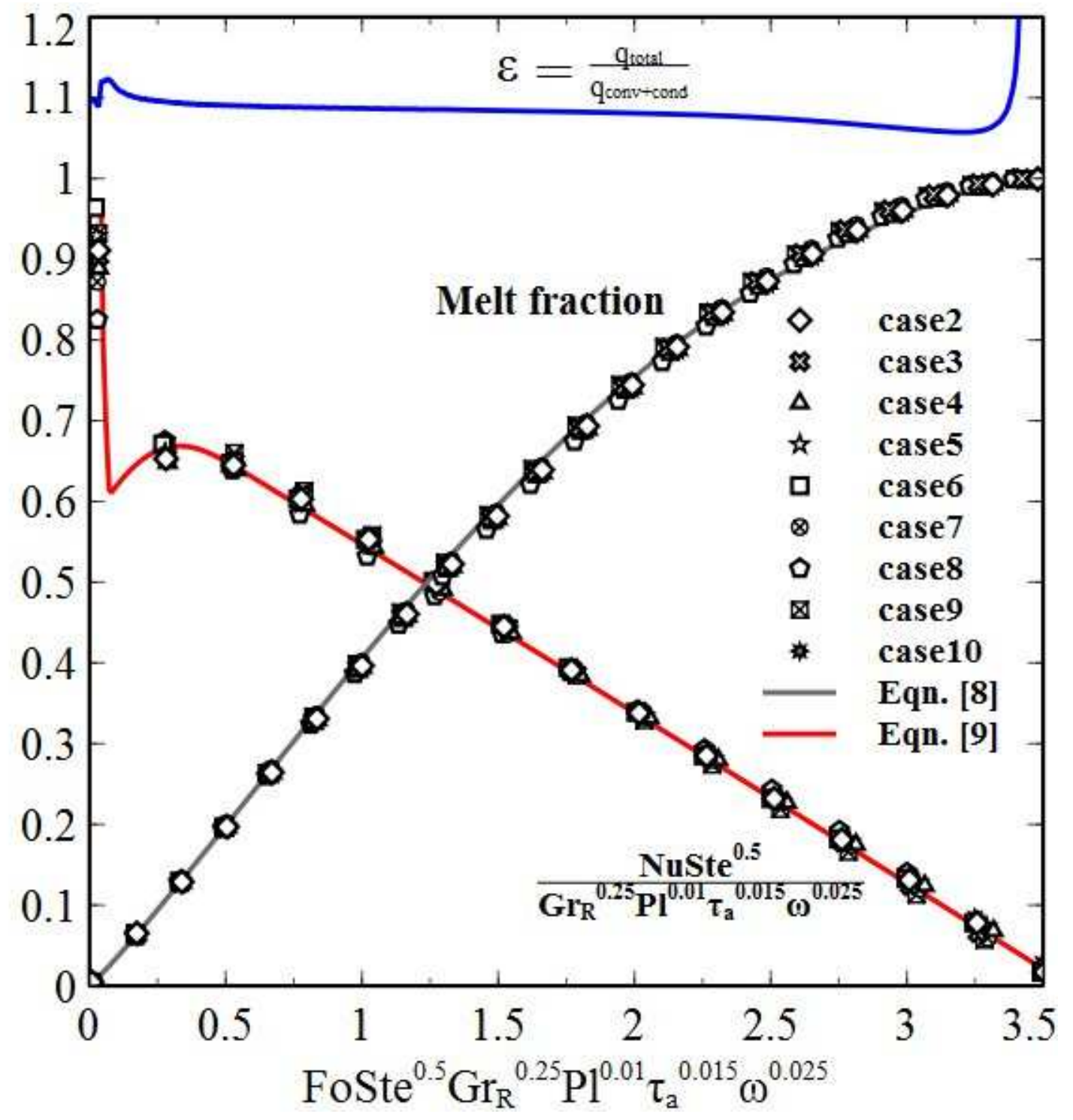

Figure 14 Melt fraction and Nusselt number as a function of the controlling parameters 
Table 1 PCM Thermo-physical properties

\begin{tabular}{|c|c|c|}
\hline Properties & Sodium Chloride & Refs. \\
\hline \multicolumn{3}{|l|}{ Density $\left(\mathrm{kg} / \mathrm{m}^{3}\right)$} \\
\hline solid phase & 2160 & {$[43]$} \\
\hline mushy zone & $1290180-1200 T$ & \\
\hline Liquid phase & $2168.1-0.5663 T$ & {$[44]$} \\
\hline Dynamic viscosity $(\mathrm{kg} / \mathrm{m} \mathrm{s})$ & $0.0034-2.194 \times 10^{-6} T$ & {$[44]$} \\
\hline Latent heat of fusion $(\mathrm{J} / \mathrm{kg})$ & 479289 & {$[45]$} \\
\hline Melting temperature $\left({ }^{\circ} \mathrm{C}\right)$ & 800.7 & {$[45]$} \\
\hline absorption coefficient $\left(m^{-1}\right)$ & $0-200$ & \\
\hline \multicolumn{3}{|l|}{ Specific heat $(J / k g K)$} \\
\hline solid phase & $1662.3-0.4218 T$ & {$[45]$} \\
\hline Liquid phase & $3289.3-3.4589 T+0.0014173 T^{2}$ & {$[45]$} \\
\hline Thermal conductivity $(\mathrm{W} / \mathrm{mK})$ & $-0.269+9.07 \times 10^{-4} T$ & {$[46]$} \\
\hline
\end{tabular}


Table 2 Dimensionless total and radiative heat fluxes

\begin{tabular}{ccccc}
\hline \hline \multirow{2}{*}{$\tau_{2}$} & \multicolumn{2}{c}{ Inner wall } & \multicolumn{2}{c}{ Outer wall } \\
\cline { 2 - 5 } & $\Psi_{1}$ & $\Phi_{1}$ & $\Psi_{2}$ & $\Phi_{2}$ \\
\hline \multirow{2}{*}{1} & $4.257^{\mathrm{a}}$ & $0.807^{\mathrm{a}}$ & $1.064^{\mathrm{a}}$ & $0.202^{\mathrm{a}}$ \\
& $4.252^{\mathrm{b}}$ & $0.805^{\mathrm{b}}$ & $1.063^{\mathrm{b}}$ & $0.201^{\mathrm{b}}$ \\
\hline \multirow{2}{*}{2} & $6.070^{\mathrm{a}}$ & $0.709^{\mathrm{a}}$ & $1.517^{\mathrm{a}}$ & $0.160^{\mathrm{a}}$ \\
& $6.076^{\mathrm{b}}$ & $0.733^{\mathrm{b}}$ & $1.519^{\mathrm{b}}$ & $0.159^{\mathrm{b}}$ \\
\hline \multirow{2}{*}{3} & $7.408^{\mathrm{a}}$ & $0.618^{\mathrm{a}}$ & $1.852^{\mathrm{a}}$ & $0.127^{\mathrm{a}}$ \\
& $7.462^{\mathrm{b}}$ & $0.620^{\mathrm{b}}$ & $1.865^{\mathrm{b}}$ & $0.129^{\mathrm{b}}$ \\
\hline \multirow{2}{*}{4} & $8.440^{\mathrm{a}}$ & $0.549^{\mathrm{a}}$ & $2.110^{\mathrm{a}}$ & $0.106^{\mathrm{a}}$ \\
& $8.511^{\mathrm{b}}$ & $0.551^{\mathrm{b}}$ & $2.128^{\mathrm{b}}$ & $0.107^{\mathrm{b}}$ \\
\hline \multirow{2}{*}{10} & $11.341^{\mathrm{a}}$ & $0.322^{\mathrm{a}}$ & $2.834^{\mathrm{a}}$ & $0.052^{\mathrm{a}}$ \\
& $11.455^{\mathrm{b}}$ & $0.319^{\mathrm{b}}$ & $2.864^{\mathrm{b}}$ & $0.052^{\mathrm{b}}$ \\
\hline
\end{tabular}

a. Predictions from the present numerical approach.

b. Values reported in Ref. [18] 
Table 3 Analyzed study cases

\begin{tabular}{ccccccccc}
\hline \hline case & $R_{i}(m)$ & $\Delta T\left({ }^{\circ} \mathrm{C}\right)$ & $\kappa_{a}$ & $\sigma_{s}$ & $\tau$ & $P l$ & $G r_{R}$ & Ste \\
\hline 1 & 0.02 & 25 & 0 & 0 & 0 & 0 & $1.7 \times 10^{6}$ & 0.063 \\
\hline 2 & 0.02 & 25 & 25 & 0 & 0.5 & 0.03 & $1.7 \times 10^{6}$ & 0.063 \\
3 & 0.02 & 25 & 100 & 0 & 2.0 & 0.13 & $1.7 \times 10^{6}$ & 0.063 \\
4 & 0.02 & 25 & 200 & 0 & 4.0 & 0.25 & $1.7 \times 10^{6}$ & 0.063 \\
\hline 5 & 0.025 & 25 & 200 & 0 & 5.0 & 0.25 & $3.3 \times 10^{6}$ & 0.063 \\
6 & 0.03 & 25 & 200 & 0 & 6.0 & 0.25 & $5.7 \times 10^{6}$ & 0.063 \\
\hline 7 & 0.02 & 20 & 100 & 0 & 2.0 & 0.13 & $1.3 \times 10^{6}$ & 0.050 \\
8 & 0.02 & 15 & 100 & 0 & 2.0 & 0.13 & $9.9 \times 10^{5}$ & 0.038 \\
\hline 9 & 0.02 & 25 & 10 & 15 & 0.5 & 0.03 & $1.7 \times 10^{6}$ & 0.063 \\
10 & 0.02 & 25 & 10 & 90 & 2.0 & 0.13 & $1.7 \times 10^{6}$ & 0.063 \\
\hline \hline
\end{tabular}

